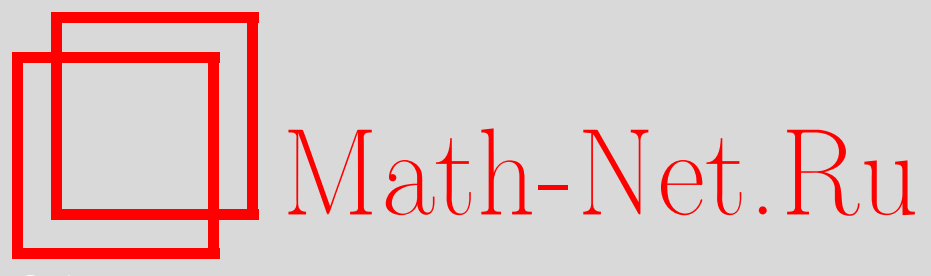

В. В. Штепин, Т. В. Штепина, Применение сплетающих операторов в функциональном анализе, Изв. РАН. Сер. матем., 2009, том 73, выпуск 6, 195-220

DOI: https://doi.org/10.4213/im2715

Использование Общероссийского математического портала Math-Net.Ru подразумевает, что вы прочитали и согласны с пользовательским соглашением http://www . mathnet.ru/rus/agreement

Параметры загрузки:

IP : 52.6 .47 .48

26 апреля 2023 г., 13:55:48

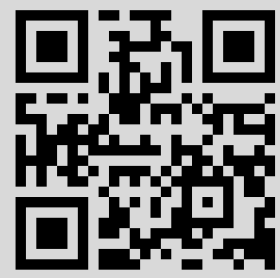


УДК 515.12

В. В. Штепин, Т. В. Штепина

\title{
Применение сплетающих операторов в функциональном анализе
}

\begin{abstract}
Рассматриваются классы интегральных операторов в пространствах квадратично интегрируемых функций на сфере и локально интегрируемых функций на пространстве Лобачевского, ядра которых зависят только от расстояния между точками соответственно в сферической и гиперболической геометрии. Указанные операторы являются сплетающими операторами квазирегулярного представления соответствующей группы Ли, что позволяет вычислить их спектры и диагонализировать сами операторы. В качестве приложений рассмотрены задача Минковского и теорема Функа-Гекке для евклидового пространства $\mathbb{R}^{n}$. Получено обобщение теоремы Функа-Гекке в случае гиперболического пространства $\mathbb{R}^{n-1,1}$ с индефинитным скалярным произведением.
\end{abstract}

Библиография: 23 наименования.

Ключевые слова: сплетающий оператор, представление простого спектра, гиперболические гармоники, непрерывный базис, обобщение теоремы Функа-Гекке.

\section{Введение}

Знаменитая лемма Шура гласит, что всякий сплетающий оператор, действующий в пространстве неприводимого комплексного представления, является скалярным ${ }^{1}$. Сплетающие операторы возникают самым естественным образом в разных областях математики. Например, если какой-то оператор зависит от элемента $g$ группы $G$, то мы вправе ожидать, что его усреднение по $G$ будет обладать какими-то "хорошими" свойствами подобно тому, как математическое ожидание случайной величины имеет не случайно "хорошие" свойства. Часто такое усреднение оказывается интегральным сплетающим оператором, действующим из одного функционального пространства в другое. Приложения сплетающих операторов в алгебре встречаются достаточно часто и не вызывают удивления (упомянем лишь классическую теорему Г. Вейля о взаимной ортогональности характеров конечномерных неприводимых представлений компактной группы). Гораздо менее известны приложения сплетающих операторов в функциональном анализе, где в действительности они наиболее эффективны.

Пусть $\mathcal{A}$ - некоторое интегральное преобразование, действующее из функционального пространства $X$ в функциональное пространство $Y$. Часто $X$ и $Y$

\footnotetext{
1Эту формулировку можно немного уточнить. Всякий сплетающий оператор, действующий из пространства одного неприводимого комплексного представления в пространство другого неприводимого комплексного представления, является либо нулевым оператором (если представления не изоморфны), либо изоморфизмом (если представления изоморфны).
}

(C) В. В. Штепин, Т. В. ШтепинА, 2009 
являются пространствами представлений одной и той же группы Ли. Уместно тогда поставить вопрос, который обычно не попадает в поле зрения специалистов по интегральным операторам. Будет ли $\mathcal{A}$ сплетающим оператором? Если ответ положительный, то для каких представлений какой группы Ли?

Цель настоящей работы - показать, что данный вопрос является не праздным, так как положительный ответ на него позволяет установить более глубокие свойства интегрального преобразования (найти спектр, провести диагонализацию) и, возможно, с их помощью максимально упростить доказательства тех или иных аналитических теорем.

В §1 мы рассматриваем классическую задачу интегральной геометрии, известную как задача Минковского. Мы доказываем здесь, что выпуклое центрально-симметричное тело в $\mathbb{R}^{n}$, все сечения которого плоскостями, проходящими через центр симметрии, равновелики, является шаром. Наш подход к решению этой задачи не является новым, зато весьма поучителен: в казалось бы чисто геометрической задаче решение достигается алгебраическими методами теории представлений. Единственность решения, как ни странно, обеспечивается тем, что квазирегулярное представление группы Ли $\mathrm{SO}(n)$ в $L^{2}\left(S^{n-1}\right)$ имеет простой спектр. Отметим, что приведенные в доказательстве теоремы 1.1 формулы (1.7) и (1.8) (выражающие базис Картана-Вейля алгебры Ли $\mathfrak{s o}(n, \mathbb{C})$ через ее образующие $\left.E_{i j}-E_{j i}\right)$ имеют и самостоятельное значение, так как могут применяться к компактным формам других комплексных простых групп Ли.

В §2 мы рассматриваем обширный класс интегральных операторов в $L^{2}\left(S^{n-1}\right)$, ядра которых зависят лишь от угла между векторами $\xi, \eta$ единичной длины. Замечательная теорема Функа-Гекке утверждает, что для таких операторов всякая поверхностная сферическая гармоника является собственным вектором. В теореме 2.1 мы приводим современную формулировку теоремы Функа-Гекке, которую ей придал А. Эрдейи [1] в 1938 г. (В классической формулировке Е. Гекке [2] рассматривал только поверхностные гармоники на $S^{2}$, а ядра интегральных операторов - в виде плоской волны). Несмотря на то, что эта теорема, видимо, неоднократно переоткрывалась, все имевшиеся до недавнего времени ее доказательства были аналитическими и довольно сложными (ср., например, [1], [3, гл. IV, § 2, п. 2.19], [4, введение, § 3.2, лемма 3.6]). Наш подход, основанный на лемме Шура, позволяет получить значительно более простое доказательство. В предыдущей работе одного из авторов [5] теорема Функа-Гекке использовалась для изучения спектра эквивариантного расширения оператора Лапласа в шаре. Аналогичная задача может быть поставлена для эквивариантного расширения волнового оператора во внутренности светового конуса. Тогда необходимо обобщение теоремы Функа-Гекке на случай гиперболического пространства $\mathbb{R}^{n-1,1}$. Этому обобщению посвящен $\S 3$.

Мы начинаем $\S 3$ с изучения спектра квазирегулярного представления группы Лоренца $\mathrm{SO}_{0}(n-1-1)$, действующего в пространстве обобщенных функций на $S_{H}$, где $S_{H}$ - пространство Лобачевского. Некоторые частные результаты (теоремы 3.1, 3.2 и следствие 3.1) могут оказаться полезными как с точки зрения теории представлений, так и с точки зрения ее приложений в кван- 
товой механике. Уже сравнение поверхностных сферических гармоник $S_{K}^{l}(\theta)$ из $\S 2$ и поверхностных гиперболических гармоник $H_{L}^{n \sigma}(\theta)$ из $\S 3$ показывает, что некомпактность группы $G$ значительно усложняет исследования, вынуждая нас строить "непрерывный” базис в пространстве квазирегулярного представления группы Лоренца. Но так как спектр этого представления тоже прост, мы можем для переноса результатов, полученных в $\S 2$, на пространство Лобачевского использовать все тот же подход, основанный на сплетающих операторах и лемме Шура. В заключение мы хотели бы выразить нашу уверенность в том, что аналогичное обобщение теоремы Функа-Гекке может быть получено для всех римановых симметрических пространств ранга 1.

\section{§ 1. Задача Минковского}

С именем Минковского связывают следующую проблему: верно ли, что единственным выпуклым центрально-симметричным телом с непрерывной границей в $\mathbb{R}^{n}$, обладающим равновеликими сечениями (всевозможными) гиперплоскостями, проходящими через центр симметрии, является шар? Для случая $n=3$ доказательство имеется, например, в [6, лекция 5, п. 1.6]. Наше доказательство (за исключением теоремы 1.1, являющейся естественным приложением картановского метода старших весов) в основном следует [6].

Пусть $\varphi_{1}, \varphi_{2}, \ldots, \varphi_{n-1}$ - углы Эйлера на единичной сфере $S^{n-1}$ в $\mathbb{R}^{n}$. Точка $x \in \mathbb{R}^{n}$ с декартовыми координатами $x_{1}, x_{2}, \ldots, x_{n}$ имеет сферические координаты $\rho, \varphi_{1}, \varphi_{2}, \ldots, \varphi_{n-1}$, которые связаны с декартовыми соотношениями

$$
\begin{gathered}
x_{1}=\rho \sin \varphi_{n-1} \cdots \sin \varphi_{2} \sin \varphi_{1}, \\
x_{2}=\rho \sin \varphi_{n-1} \cdots \sin \varphi_{2} \cos \varphi_{1}, \\
\cdots \cdots \cdots \cdots \cdots \cdots \cdots \cdots \cdots \cdots \cdots \cdots \cdots \cdots \cdots \\
x_{n-1}=\rho \sin \varphi_{n-1} \cos \varphi_{n-2}, \\
x_{n}=\rho \cos \varphi_{n-1},
\end{gathered}
$$

где $\rho=|x| \geqslant 0,0 \leqslant \varphi_{1}<2 \pi, 0 \leqslant \varphi_{i}<\pi, i=2,3, \ldots, n-1$ [7, с. 415]. Будем считать, что поверхность тела $B$ задается в сферических координатах (1.1) непрерывной неотрицательной функцией $r=r\left(\varphi_{1}, \varphi_{2}, \ldots, \varphi_{n-1}\right)$. Согласно условию имеем $r=r\left(\varphi_{1}, \varphi_{2}, \ldots, \varphi_{n-1}\right)=r\left(\bar{\varphi}_{1}, \bar{\varphi}_{2}, \ldots, \bar{\varphi}_{n-1}\right)$, где $\bar{\varphi}_{i}-$ эйлеровы координаты точки сферы $S^{n-1}$, диаметрально противоположной точке с координатами $\varphi_{i}, i=1,2, \ldots, n-1$.

Пусть

$$
d \xi=\sin ^{n-2} \varphi_{n-1} \sin ^{n-3} \varphi_{n-2} \cdots \sin \varphi_{2} d \varphi_{1} d \varphi_{2} \ldots d \varphi_{n-1}
$$

- инвариантная относительно вращений из группы $\mathrm{SO}(n)$ мера на сфере $S^{n-1}$, нормированная условием

$$
\int_{S^{n-1}} d \xi=\mu\left(S^{n-1}\right)=\frac{2 \pi^{\frac{n}{2}}}{\Gamma\left(\frac{n}{2}\right)},
$$


где $\mu\left(S^{n-1}\right)$ - площадь поверхности единичной сферы $S^{n-1}$. В пространстве $L^{2}\left(S^{n-1}\right)$ действует квазирегулярное представление $T$ группы $\mathrm{SO}(n)$, определяемое равенством

$$
[T(g) f](\xi)=f\left(g^{-1} \xi\right), \quad \xi \in S^{n-1}, \quad g \in \mathrm{SO}(n) .
$$

Это представление унитарно относительно скалярного произведения

$$
\left(f_{1}, f_{2}\right)=\int_{S^{n-1}} f_{1}(\xi) \overline{f_{2}(\xi)} d \xi .
$$

Хорошо известно, что квазирегулярное представление $T$ приводимо. Опишем его спектр с точки зрения теории представлений групп.

Пусть $\mathfrak{R}^{n, l}$ - пространство (над полем $\mathbb{C}$ ) однородных гармонических многочленов степени $l$ от $n$ переменных. Если $f(x) \in \mathfrak{R}^{n, l}$, то его сужение на сферу $S^{n-1}$ называется поверхностной сферической гармоникой степени $l$ и обозначается через $f(\xi), \xi \in S^{n-1}$. Соотношения между $f(x)$ и $f(\xi)$ вытекает из условия однородности:

$$
f(x)=f(r \cdot \xi)=r^{l} f(\xi), \quad r=|x| .
$$

Поверхностные сферические гармоники степени $l$, очевидно, также образуют линейное пространство над $\mathbb{C}$, мы будем его обозначать $\mathcal{R}^{n, l}$. Очевидно включение $\mathcal{R}^{n, l} \subset L^{2}\left(S^{n-1}\right)$. Операторы квазирегулярного представления (1.2) переводят однородные гармонические многочлены в однородные гармонические (с сохранением степени), поэтому $\mathcal{R}^{n, l}$ является инвариантным подпространством в $L^{2}\left(S^{n-1}\right)$. Его размерность определяется формулой

$$
\operatorname{dim} \mathcal{R}^{n, l}=\frac{(n+l-3) !(n+2 l-2)}{l !(n-2) !}
$$

Известно также, что подпространства $\mathcal{R}^{n, l}$ являются неприводимыми взаимно ортогональными относительно (1.3) прямыми слагаемыми квазирегулярного представления $T$ [7, с. 424], [3, с. 160].

Пусть $P_{k}$ - множество старших весов группы Ли $\mathrm{SO}(n), k=[n / 2]$.

ТеОрема 1.1. Представление $T^{l}$ группы Ли $\mathrm{SO}(n)$ в пространстве $\mathcal{R}^{n, l}$ nоверхностных сферических гармоник степени $l$ oт $n$ переменных изоморфно неприводимому представлению $T_{(l, 0, \ldots, 0)}$ со старшим весом $(l, 0, \ldots, 0) \in P_{k}$.

ДокАзАтЕльство. Перейдем от представлений группы Ли $\mathrm{SO}(n)$ к представлениям соответствующей алгебры Ли $\mathfrak{s o}(n)$ и воспользуемся методом старших весов Картана.

Алгебра Ли $\mathfrak{s o}(n)$ имеет стандартный базис, порожденный кососимметрическими матрицами

$$
X_{i j}=E_{i j}-E_{j i}, \quad 1 \leqslant i<j \leqslant n,
$$

где $E_{i j}$ - матрица, имеющая единственный ненулевой элемент 1 на пересечении $i$-й строки и $j$-го столбца. Матрицы $X_{i j}$ удовлетворяют следующим соотношениям коммутации:

$$
\left[X_{i j}, X_{k l}\right]=\delta_{j k} X_{i l}+\delta_{i l} X_{j k}-\delta_{i k} X_{j l}-\delta_{j l} X_{i k} .
$$


При использовании равенства (1.5) следует иметь в виду, что

$$
X_{p q}=-X_{q p}
$$

Как известно, $\mathrm{SO}(n)$ является компактной формой комплексной простой группы Ли $\mathrm{SO}(n, \mathbb{C}), n \geqslant 3$, причем представление $T^{l}$ неприводимо тогда и только тогда, когда неприводимо его аналитическое комплексное продолжение на группу $\mathrm{SO}(n, \mathbb{C})[8$, с. 527]. Поэтому в дальнейшем мы будем рассматривать действие именно группы Ли $\mathrm{SO}(n, \mathbb{C})$ и ее алгебры Ли $\mathfrak{s o}(n, \mathbb{C})$ в $\mathcal{R}^{n, l}$. В алгебре Ли $\mathfrak{s o}(n, \mathbb{C})$ удобнее выбирать не базис $(1.4)$, а так называемый базис Картана-Вейля, состоящий из векторов, собственных относительно картановской ${ }^{2}$ подалгебры $\mathfrak{h}$. Пусть элементы алгебры $\mathfrak{s o}(n, \mathbb{C})$ реализованы линейными операторами, действующими в пространстве $\mathbb{C}^{2 k}$ (при $n=2 k$ ) с базисом $e_{1}, \ldots, e_{k}, e_{-k}, \ldots, e_{-1}$ или в $\mathbb{C}^{2 k+1}$ (при $\left.n=2 k+1\right)$ с базисом $e_{1}, \ldots, e_{k}, e_{0}, e_{-k}, \ldots, e_{-1}$. Тогда если $n$ четно, то $\mathfrak{s o}(n, \mathbb{C})$ порождается операторами

$$
A_{11}=i X_{1,-1}, \quad A_{22}=i X_{2,-2}, \quad \ldots, \quad A_{k k}=i X_{k,-k},
$$

являющимися образующими подалгебры Картана $\mathfrak{h}$, и операторами

$$
\begin{aligned}
A_{i j}=\frac{i}{2}\left(X_{i j}-X_{-j,-i}+i X_{i,-j}+i X_{j,-i}\right), & 0<i<|j|, \\
A_{j i}=-\frac{i}{2}\left(X_{j i}-X_{-i,-j}-i X_{-i, j}-i X_{-j, i}\right), & 0<i<|j| .
\end{aligned}
$$

Если $n$ нечетно, то помимо образующих (1.7), (1.8) базис Картана-Вейля содержит также образующие

$$
A_{i 0}=\frac{1}{2}\left((1+i) X_{i 0}+(-1+i) X_{0,-i}\right), \quad A_{0 i}=\frac{1}{2}\left((-1+i) X_{i 0}+(1+i) X_{0,-i}\right),
$$

где $i=1,2, \ldots, k$.

Отметим, что образующие (1.8) не являются независимыми. Легко проверить, применяя равенства (1.6), что они удовлетворяют соотношениям

$$
A_{-j,-i}=-A_{i j}
$$

Независимыми образующими вида (1.8) являются $A_{i j}, A_{j i}$, где $0<i<|j|$. Легко видеть, что образующие (1.7) являются собственными для $\mathfrak{h}$ с собственными значениями

$$
(0, \ldots, 0, \pm 1,0, \ldots, 0, \mp \operatorname{sign}(j), \ldots, 0),
$$

где верхние (соответственно, нижние) знаки используются для $A_{i j}$ (соответственно, $\left.A_{j i}\right), 0<i<|j|$; ненулевые собственные значения находятся на

\footnotetext{
${ }^{2}$ Картановской подалгеброй $\mathfrak{h}$ в полупростой алгебре Ли $\mathfrak{g}$ называется максимальная коммутативная подалгебра, совпадающая со своим нормализатором. Говоря о собственных векторах $\mathfrak{g}$ относительно $\mathfrak{h}$, мы подразумеваем, что $\mathfrak{h}$ действует в $\mathfrak{g}$ посредством присоединенного представления.
} 
местах $i$ и $|j|$. В свою очередь, образующие $A_{i 0}$ (соответственно, $\left.A_{0 i}\right)$ имеют относительно $\mathfrak{h}$ собственные значения $(0, \ldots, 0,1,0, \ldots, 0)$ (соответственно, $(0, \ldots, 0,-1,0, \ldots, 0))$ с единственным ненулевым элементом на $i$-м месте.

Проводя несложные вычисления с учетом соотношений (1.5) и (1.6), получаем, что образующие $A_{i j}$ удовлетворяют следующим привычным соотношениям коммутации, присущим алгебре $\mathfrak{s o}(n, \mathbb{C})$ :

$$
\left[A_{i j}, A_{k l}\right]=\delta_{k j} A_{i l}-\delta_{i l} A_{k j}+\delta_{-l, j} A_{k,-i}-\delta_{k-i} A_{-j, l}
$$

(cp. с [9], [10]).

Рассмотрим многочлен $f_{0}\left(x_{1}, \ldots, x_{n}\right)=\left(x_{n}+i x_{1}\right)^{l}$, или, в обозначениях координат переменными с индексами, принадлежащими $\{0 ; \pm 1 ; \ldots ; \pm k\}$, многочлен

$$
f_{0}(x)=\left(x_{-1}+i x_{1}\right)^{l} .
$$

Легко видеть, что $f_{0}(x)$ является однородным гармоническим многочленом степени $l$, т. е. принадлежит пространству $\mathfrak{R}^{n, l}$.

Наша дальнейшая цель - показать, что $\left.f_{0}(x)\right|_{S^{n-1}}$ является старшим вектором представления $T^{l}$ группы Ли $\mathrm{SO}(n, \mathbb{C})$ в пространстве $\mathcal{R}^{n, l}$, или, что равносильно, $f_{0}(x)$ является старшим вектором представления $\mathrm{SO}(n, \mathbb{C})$ в пространстве $\mathfrak{R}^{n, l}$. Определим, как обычно, дифференциал квазирегулярного представления $T^{l}$ в $\mathfrak{R}^{n, l}$ равенством

$$
\left[\tilde{A}_{i j} f\right](x)=\left.\frac{d}{d t} f\left(\exp \left(-t A_{i j}\right) x\right)\right|_{t=0}, \quad f \in \mathfrak{R}^{n, l} .
$$

Тогда соответствие $A_{i j} \mapsto \tilde{A}_{i j}$ задает представление алгебры Ли $\mathfrak{s o}(n, \mathbb{C})$ в том же самом пространстве [8, с. 144]. Теперь ввиду очевидного представления

$$
\widetilde{X}_{i j}=x_{i} \frac{\partial}{\partial x_{j}}-x_{j} \frac{\partial}{\partial x_{i}}
$$

для рассмотренных выше базисных векторов $\mathfrak{s o}(n, \mathbb{C})$ будем иметь

$$
\begin{gathered}
\tilde{A}_{s s}=i\left(x_{s} \frac{\partial}{\partial x_{-s}}-x_{-s} \frac{\partial}{\partial x_{s}}\right), \quad s=1,2, \ldots, k, \\
\tilde{A}_{p q}=\frac{1}{2}\left(x_{p} \frac{\partial}{\partial x_{q}}-x_{q} \frac{\partial}{\partial x_{p}}+x_{-p} \frac{\partial}{\partial x_{-q}}-x_{-q} \frac{\partial}{\partial x_{-p}}\right. \\
\left.+i\left(x_{p} \frac{\partial}{\partial x_{-q}}-x_{-q} \frac{\partial}{\partial x_{p}}+x_{q} \frac{\partial}{\partial x_{-p}}-x_{-p} \frac{\partial}{\partial x_{q}}\right)\right),
\end{gathered}
$$

где $0<p<|q|$, и (в случае нечетного $n$ )

$$
\begin{aligned}
& \tilde{A}_{p 0}=\frac{1+i}{2}\left(x_{p} \frac{\partial}{\partial x_{0}}-x_{0} \frac{\partial}{\partial x_{p}}\right)+\frac{-1+i}{2}\left(x_{0} \frac{\partial}{\partial x_{-p}}-x_{-p} \frac{\partial}{\partial x_{0}}\right), \\
& \tilde{A}_{0 p}=\frac{-1+i}{2}\left(x_{p} \frac{\partial}{\partial x_{0}}-x_{0} \frac{\partial}{\partial x_{p}}\right)+\frac{1+i}{2}\left(x_{0} \frac{\partial}{\partial x_{-p}}-x_{-p} \frac{\partial}{\partial x_{0}}\right),
\end{aligned}
$$

где $p=1,2, \ldots, k$. 
Рассмотренный выше многочлен $f_{0}$ является собственным относительно картановской подалгебры $\mathfrak{h}$ и аннулируется положительными корневыми векторами $\mathfrak{s o}(n, \mathbb{C})$ :

$$
\begin{gathered}
\tilde{A}_{11} f_{0}=l f_{0}, \quad \tilde{A}_{s s} f_{0}=0, \quad s=2,3, \ldots, k, \\
\tilde{A}_{p q} f_{0}=0, \quad 0<p<|q|,
\end{gathered}
$$

и в случае нечетного $n$

$$
\tilde{A}_{p 0} f_{0}=0, \quad p=1,2, \ldots, k .
$$

Следовательно, $f_{0}(x)$ является старшим вектором представления $T_{(l, 0, \ldots, 0)} \subseteq$ $\mathfrak{R}^{n, l}$ со старшим весом $(l, 0, \ldots, 0) \in P_{k}$ группы Ли $\mathrm{SO}(n)$. Для завершения доказательства остается заметить, что

$$
\operatorname{dim} T_{(l, 0, \ldots, 0)}^{\mathrm{SO}(n)}=\frac{(n+l-3) !(n+2 l-2)}{l !(n-2) !}=\operatorname{dim} \Re^{n, l}
$$

(ср. [11, с. 178-180]). Теорема доказана.

Пусть $\nu=\left(\nu_{1}, \nu_{2}, \ldots, \nu_{n}\right)$ - единичный вектор в $\mathbb{R}^{n}$, и пусть $\varphi_{1}, \varphi_{2}, \ldots, \varphi_{n}-$ соответствующие вектору $\nu$ значения углов Эйлера. Отметим, что вектору $\nu$ отвечает набор эйлеровых координат $\varphi=\left(\varphi_{1}, \varphi_{2}, \ldots, \varphi_{n}\right), 0 \leqslant \varphi_{1}<2 \pi$, $0 \leqslant \varphi_{i}<\pi, i=2,3, \ldots, n-1$, и будем применять краткое обозначение $\nu=\nu(\varphi)$. Связи между этими параметрами точки единичной сферы выражаются формулами

$$
\cos \varphi_{i}=\frac{\nu_{i+1}}{r_{i+1}}, \quad \sin \varphi_{i}=\frac{r_{i}}{r_{i+1}}
$$

где $r_{i}^{2}=\nu_{1}^{2}+\cdots+\nu_{i}^{2}, i=1,2, \ldots, n-1, r_{n}^{2}=|\nu|=1$. Далее мы будем отождествлять точку $\nu(\varphi)$ на сфере $S^{n-1}$ с набором $\varphi$ ее эйлеровых координат.

Обозначим через $C\left(\varphi_{1}, \varphi_{2}, \ldots, \varphi_{n-1}\right)$ и $B\left(\varphi_{1}, \varphi_{2}, \ldots, \varphi_{n-1}\right)$ сечения соответственно единичной сферы $S^{n-1}$ и тела $B$ гиперплоскостью с вектором нормали $\nu(\varphi)$ :

$$
\nu_{1} x_{1}+\nu_{2} x_{2}+\cdots+\nu_{n} x_{n}=0 .
$$

ОПРЕДЕЛЕниЕ 1.1. Определим оператор $\mathcal{A}$ в $L^{2}\left(S^{n-1}\right)$ равенством

$$
[\mathcal{A} f]\left(\theta_{1}, \ldots, \theta_{n-1}\right)=\int_{C\left(\theta_{1}, \ldots, \theta_{n-1}\right)} f(\xi) d \xi, \quad f \in L^{2}\left(S^{n-1}\right) .
$$

Отметим, что вектору $\nu$ и вектору $-\nu$ отвечает одна и та же гиперплоскость (1.13), поэтому функция $\mathcal{A} f$ является четной, т. е. принимает в диаметрально противоположных точках сферы $S^{n-1}$ одинаковые значения:

$$
[\mathcal{A} f](\theta)=[\mathcal{A} f](\bar{\theta})
$$

где $\theta$ и $\bar{\theta}$ - эйлеровы координаты векторов $\varphi(\theta)$ и $-\varphi(\theta)$ соответственно.

ПредЛожениЕ 1.1. Оператор $\mathcal{A}$ является сплетающим оператором квазирегулярного представления $T$ группы Ли $\mathrm{SO}(n)$ в себя. 
ДокАЗАТЕЛЬСтво. Пусть $g$ - произвольный элемент группы $\mathrm{SO}(n)$. Тогда

$$
([\mathcal{A} \circ T(g)] f)(\theta)=\int_{C\left(\theta_{1}, \ldots, \theta_{n-1}\right)}[T(g) f](\xi) d \xi=\int_{C\left(\theta_{1}, \ldots, \theta_{n-1}\right)} f\left(g^{-1} \xi\right) d \xi .
$$

Сделаем замену переменной $g^{-1} \xi=\chi$. В силу $\mathrm{SO}(n)$-инвариантности меры имеем $d \xi=d \chi$, поэтому

$$
([\mathcal{A} \circ T(g)] f)(\theta)=\int_{g^{-1} C\left(\theta_{1}, \ldots, \theta_{n-1}\right)} f(\chi) d \chi .
$$

С другой стороны,

$$
([\mathcal{A} \circ T(g)] f)(\theta)=(\mathcal{A} f)\left(g^{-1} \theta\right)=\int_{g^{-1} C\left(\theta_{1}, \ldots, \theta_{n-1}\right)} f(\xi) d \xi .
$$

Мы воспользовались тем, что ортогональные преобразования сохраняют углы между векторами, поэтому если $\mu=g^{-1}(\nu)$ для единичных векторов $\mu$ и $\nu$, то гиперплоскость $\sum \nu_{i} x_{i}=0$ переходит под действием $g^{-1}$ в гиперплоскость $\sum \mu_{i} x_{i}=0$. Предложение доказано.

ПрЕДЛОЖЕНИЕ 1.2. Пустъ $r_{B}\left(\theta_{1}, \ldots, \theta_{n-2}\right)=\left.r\left(\varphi_{1}, \ldots, \varphi_{n-1}\right)\right|_{B\left(\psi_{1}, \ldots, \psi_{n-1}\right)}-$ радиус-вектор точки, описывающии граничу сечения $B\left(\psi_{1}, \psi_{2}, \ldots, \psi_{n-1}\right)$; $\theta_{1}, \theta_{2}, \ldots, \theta_{n-2}$ - эйлеровы координаты на сфере $C\left(\psi_{1}, \psi_{2}, \ldots, \psi_{n-1}\right) \simeq S^{n-2}$. Тогда мера сечения $B\left(\psi_{1}, \psi_{2}, \ldots, \psi_{n-1}\right)$ выражсается функиией

$$
\mu B\left(\psi_{1}, \psi_{2}, \ldots, \psi_{n-1}\right)=\frac{1}{n-1}\left[\mathcal{A} r^{n-1}\right]\left(\psi_{1}, \psi_{2}, \ldots, \psi_{n-1}\right) .
$$

ДокАзАТЕЛЬство. Пусть $|J|$ - якобиан перехода от декартовых координат к сферическим в $(n-1)$-мерной гиперплоскости, содержащей сечение $B\left(\psi_{1}, \psi_{2}, \ldots, \psi_{n-1}\right)$. Тогда имеем

$$
\begin{aligned}
\mu B\left(\psi_{1}, \ldots, \psi_{n-1}\right) & =\int_{0}^{2 \pi} \int_{0}^{\pi} \cdots \int_{0}^{\pi} \int_{0}^{r_{B}\left(\theta_{1}, \ldots, \theta_{n-2}\right)}|J| d \rho d \theta_{n-2} \ldots d \theta_{2} d \theta_{1} \\
& =\int_{0}^{2 \pi} \int_{0}^{\pi} \cdots \int_{0}^{\pi} \int_{0}^{r_{B}\left(\theta_{1}, \ldots, \theta_{n-2}\right)} \rho^{n-2} d \rho d \xi \\
& =\frac{1}{n-1} \int_{0}^{2 \pi} \int_{0}^{\pi} \cdots \int_{0}^{\pi} r_{B}^{n-1}\left(\theta_{1}, \ldots, \theta_{n-2}\right) d \xi
\end{aligned}
$$

Здесь, как и выше, $d \xi=\sin ^{n-3} \theta_{n-2} \cdot \sin ^{n-4} \theta_{n-3} \cdots \sin \theta_{2} d \theta_{n-2} \ldots d \theta_{1}-$ мера на единичной сфере $C\left(\psi_{1}, \psi_{2}, \ldots, \psi_{n-1}\right) \simeq S^{n-2}$, инвариантная относительно вращений из группы $\mathrm{SO}(n)$.

Теперь согласно определению 1.1 имеем

$$
\begin{aligned}
\mu B\left(\psi_{1}, \ldots, \psi_{n-1}\right) & =\frac{1}{n-1} \int_{C\left(\psi_{1}, \ldots, \psi_{n-1}\right)} r_{B}^{n-1}\left(\theta_{1}, \ldots, \theta_{n-2}\right) d \xi \\
& =\frac{1}{n-1}\left[\mathcal{A} r^{n-1}\right]\left(\psi_{1}, \ldots, \psi_{n-1}\right) .
\end{aligned}
$$

Предложение доказано. 
Отметим, что согласно (1.15) мера сечения $\mu B\left(\psi_{1}, \ldots, \psi_{n-1}\right)$ является образом оператора $\mathcal{A}$ от четной на сфере $S^{n-1}$ функции $r^{n-1}$, так как по условию задачи Минковского $r(\varphi)=r(\bar{\varphi})$.

ПРЕДЛОЖЕНИЕ 1.3. На каждом из инвариантных подпространств $R^{n, l}$ оператор $\mathcal{A}$ является скалярным оператором с собственным значением $\lambda_{n, l}$, определяемым равенствами

$$
\lambda_{n, l}= \begin{cases}\frac{2 \pi^{\frac{n-1}{2}}}{\Gamma\left(\frac{n-1}{2}\right)} \frac{C_{l}^{\frac{n-2}{2}}(0)}{C_{l}^{\frac{n-2}{2}}(1)}, & \text { если l четно } \\ 0, & \text { если l нечетно }\end{cases}
$$

где $C_{l}^{p}(t)-$ многочлены Гегенбауэра.

ДокАзАтельство. Квазирегулярное представление $T$ в $L^{2}\left(S^{n-1}\right)$ согласно теореме 1.1 имеет простой спектр. Поскольку всякий сплетающий оператор неприводимого представления в неприводимое является либо изоморфизмом, либо нулевым оператором, то $\mathcal{A}$ может переводить каждое подпространство $R^{n, l}$ только в себя. Теперь согласно лемме Шура оператор $\mathcal{A}$ на подпространстве $R^{n, l}$ является скалярным. Для вычисления соответствующего собственного значения $\lambda_{n, l}$ возьмем в качестве тестовой функции зональную сферическую гармонику

$$
\varphi_{n, l}(x)=\frac{C_{l}^{\frac{n-2}{2}}(\xi)}{C_{n}^{\frac{n-2}{2}}(1)} \in R^{n, l}
$$

(см. [7, с. $437,(6)])$, где $x=\left(x_{1}, \ldots, x_{n-1}, \xi\right) \in S^{n-1}$, а $C_{l}^{p}(t)$ - многочлены Гегенбауэра. Отметим, что если $\nu_{0}=(0, \ldots, 0,1)$ - "северный полюс" сферы $S^{n-1}$, то его эйлеровы координаты можно считать равными нулю: $\left(\theta_{1}, \ldots, \theta_{n-1}\right)=$ $(0, \ldots, 0)$. (В действительности только последняя координата $\theta_{n-1}$ полюса $\nu_{0}$ определена однозначно, как это следует из (1.12).) Зональная сферическая гармоника инвариантна относительно вращений из подгруппы $\mathrm{SO}(n-1)$, сохраняющих координату $x_{n}$, поэтому для $\nu_{0}$ сфера интегрирования $C(0,0, \ldots, 0)$ целиком принадлежит поверхности уровня функции $\varphi_{n, l}(x)$. Теперь если $l$ нечетно, то $\mathcal{A} \varphi_{n, l} \equiv 0$ по определению 1.1 ввиду равенства $\varphi_{n, l}(\theta)=-\varphi_{n, l}(\bar{\theta})$, а при четных $l$ имеем

$$
\begin{aligned}
{\left[\mathcal{A} \varphi_{n, l}\right]\left(\nu_{0}\right) } & =\int_{C(0,0, \ldots, 0)} \varphi_{n, l}(\xi) d \xi=\left.\mu(C(0,0, \ldots, 0)) \varphi_{n, l}(x)\right|_{x_{n}=0} \\
& =\mu\left(S^{n-2}\right) \frac{C_{l}^{\frac{n-2}{2}}(0)}{C_{l}^{\frac{n-2}{2}}(1)} \varphi_{n, l}\left(\nu_{0}\right)=\frac{2 \pi^{\frac{n-1}{2}}}{\Gamma\left(\frac{n-1}{2}\right)} \frac{C_{l}^{\frac{n-2}{2}}(0)}{C_{l}^{\frac{n-2}{2}}(1)} \varphi_{n, l}\left(\nu_{0}\right) .
\end{aligned}
$$

Предложение доказано.

СледСтвиЕ 1.1. Оператор $\mathcal{A}$ невырожден на подпространстве $\bigoplus_{k=0}^{\infty} R^{n, 2 k}$. 
Теорема 1.2 (о тривиальности решения задачи Минковского в $\mathbb{R}^{n}$ ). Шар единственное выпуклое центрально-симметричное тело с непрерывной гранищей в $\mathbb{R}^{n}$, имеющее равновеликие сечения гиперплоскостями, проходящими через чентр симметрии.

ДокАЗАТЕЛЬство. Пусть все сечения тела $B$ указанными гиперплоскостями имеют меру $\mu$. Тогда согласно предложению 1.2 имеем

$$
\left[\mathcal{A} r^{n-1}\right]\left(\psi_{1}, \psi_{2}, \ldots, \psi_{n-1}\right) \equiv(n-1) \mu
$$

С другой стороны, для шара $B_{R}$ с радиусом

$$
R=\frac{1}{\sqrt{\pi}}\left[\frac{n-1}{2} \Gamma\left(\frac{n-1}{2}\right) \mu\right]^{\frac{1}{n-1}}
$$

имеем

$$
\left[\mathcal{A} R^{n-1}\right]\left(\psi_{1}, \psi_{2}, \ldots, \psi_{n-1}\right) \equiv R^{n-1} \mu\left(S^{n-2}\right) \equiv(n-1) \mu
$$

откуда

$$
\left[\mathcal{A}\left(r^{n-1}-R^{n-1}\right)\right]\left(\psi_{1}, \psi_{2}, \ldots, \psi_{n-1}\right) \equiv 0 .
$$

Однако функция $r^{n-1}-R^{n-1}$ в силу условия центральной симметричности тела $B$ является четной непрерывной функцией на сфере $S^{n-1}$, а значит, принадлежит пространству $\bigoplus_{k=0}^{\infty} R^{n, 2 k}$. Действительно, по теореме Вейерштрасса всякая непрерывная функция на компакте может быть равномерно приближена многочленами. Теперь по следствию 1.1 имеем $r\left(\psi_{1}, \ldots, \psi_{n-1}\right) \equiv R$. Теорема доказана.

\section{§ 2. Простое доказательство теоремы Функа-Гекке}

Теорема Функа-Гекке утверждает, что для интегральных операторов, ядра которых зависят лишь от угла между векторами $\xi$ и $\eta$, концы которых лежат на сфере интегрирования, всякая поверхностная сферическая гармоника является собственным вектором. Эта теорема берет начало от работы П. Функа [12], в которой подробно изучались поверхностные сферические гармоники на двумерной сфере $S^{2}$. Е. Гекке [2], опираясь на работу П. Функа, сформулировал теорему о том, что поверхностные сферические гармоники являются собственными векторами интегральных операторов, ядра которых являются гладкими функциями от расстояния $\rho$ между точками $\xi$ и $\eta$ на поверхности сферы в сферической тригонометрии. В современной формулировке эта теорема появилась в работе А. Эрдейи [1], который, с одной стороны, развил утверждение теоремы до общего случая сферы $S^{n-1} \subset \mathbb{R}^{n}$, а с другой - ослабил требования, налагаемые на ядро интегрального оператора. Согласно [1] ядро может быть функцией, абсолютно интегрируемой по Лебегу вместе с квадратом. Впрочем, как показал В. С. Владимиров [13], достаточно лишь, чтобы ядро было функцией из $L^{1}(-1,1)$. 
Прежде чем формулировать эту теорему, напомним, что в пространстве $\mathcal{R}^{n, l}$ поверхностные сферические гармоники $S_{K}^{l}(\theta)$ образуют ортогональный базис:

$$
S_{K}^{l}(\theta)=\prod_{j=0}^{n-3} C_{m_{j}-m_{j+1}}^{\frac{n}{2}-\frac{j}{2}-1+m_{j+1}} \cos \left(\theta_{n-j-1}\right) \sin ^{m_{j+1}}\left(\theta_{n-j-1}\right) e^{ \pm i m_{n-2} \theta_{1}},
$$

где $\theta_{1}, \theta_{2}, \ldots, \theta_{n-1}$ - углы Эйлера на сфере $S^{n-1}, l=m_{0}, K=\left(m_{1}, \ldots, m_{n-3}\right.$, $\left.\pm m_{n-2}\right)$, причем $m_{0} \geqslant m_{1} \geqslant \cdots \geqslant m_{n-3} \geqslant m_{n-2} \geqslant 0$ [7, с. 444-445]. Ортогональность сферических гармоник означает, что $S_{K}^{l}(\theta)$ и $S_{M}^{t}(\theta)$ ортогональны друг другу относительно скалярного произведения (1.3), если $l \neq t$ или мультииндексы $K=\left(m_{1}, m_{2}, \ldots, m_{n-3}, \pm m_{n-2}\right)$ и $M=\left(m_{1}^{\prime}, m_{2}^{\prime}, \ldots, m_{n-3}^{\prime}, \pm m_{n-2}^{\prime}\right)$ не совпадают.

Теорема 2.1 (Функа-Гекке). Пусть $F(x)$ - функиия действительного переменного $x$, абсолютно интегрируемая по Лебегу вместе с квадратом на $[-1,1]$, и пусть $S_{K}^{l}(\xi)$ - любая поверхностная сферическая гармоника степени l. Тогда для любого единичного вектора $\eta$ имеет место равенство

$$
\int_{S^{n-1}(\xi)} F[(\xi, \eta)] S_{K}^{l}(\xi) d \xi=\lambda_{n, l} S_{K}^{l}(\eta)
$$

¿əe

$$
\lambda_{n, l}=i^{l}(2 \pi)^{\frac{n}{2}} \int_{-\infty}^{+\infty} t^{\frac{2-n}{2}} J_{l-1+\frac{n}{2}}(t) f(t) d t, \quad f(t)=\frac{1}{2 \pi} \int_{-1}^{1} e^{-i x t} F(x) d x .
$$

Как и при решении задачи Минковского, идея простого доказательства этой теоремы состоит в правильном введении интегрального оператора $\mathcal{A}$ : $L^{2}\left(S^{n-1}\right) \rightarrow L^{2}\left(S_{n-1}\right)$, который будет сплетающим оператором квазирегулярного представления $T$ группы Ли $\mathrm{SO}(n)$, и затем воспользоваться леммой Шура.

ОПРЕДЕЛЕНИЕ 2.1. Пусть функция $F(x)$ удовлетворяет условиям теоремы 2.1. Определим оператор $\mathcal{A}$ в $L^{2}\left(S^{n-1}\right)$ равенством

$$
[\mathcal{A} f](\eta)=\int_{S^{n-1}(\xi)} F[(\xi, \eta)] f(\xi) d \xi
$$

В следующем предложении сформулировано общее свойство всех интегральных операторов вида (2.4), ядра которых зависят от скалярного произведения $(\xi, \eta)$.

ПРЕДЛОЖЕнИЕ 2.1. Введенный формулой (2.4) интегралъный оператор $\mathcal{A}$ : $L^{2}\left(S^{n-1}\right) \rightarrow L^{2}\left(S^{n-1}\right)$ является сплетающим оператором квазирегулярного представления $T$ группы $\mathrm{SO}(n)$.

ДокАЗАтЕЛьСтво. Пусть $g$ - произвольный элемент группы $\mathrm{SO}(n)$ и $\eta-$ вектор единичной длины. Тогда для любой функции $f \in L^{2}\left(S^{n-1}\right)$ выполнено

$$
\begin{aligned}
{[\mathcal{A} \circ T(g) f](\eta) } & =\mathcal{A}(T(g) f)(\eta)=\int_{S^{n-1}(\xi)} F[(\xi, \eta)](T(g) f)(\xi) d \xi \\
& =\int_{S^{n-1}(\xi)} F[(\xi, \eta)] f\left(g^{-1} \xi\right) d \xi .
\end{aligned}
$$


Сделаем замену переменной $g^{-1} \xi=\chi$ и воспользуемся инвариантностью меры $d \xi$ относительно ортогональных вращений. Тогда

$$
\begin{aligned}
{[\mathcal{A} \circ T(g) f](\eta) } & =\int_{S^{n-1}(g \chi)} F[(g \chi, \eta)] f(\chi) d(g \chi)=\int_{S^{n-1}(\chi)} F[(g \chi, \eta)] f(\chi) d(\chi) \\
& =\int_{S^{n-1}(\chi)} F\left[\left(\chi, g^{-1} \eta\right)\right] f(\chi) d(\chi)=[\mathcal{A} f]\left(g^{-1} \eta\right)=[T(g) \circ \mathcal{A} f](\eta) .
\end{aligned}
$$

Итак, для всех $g \in \mathrm{SO}(n)$ справедливо $\mathcal{A} \circ T(g)=T(g) \circ \mathcal{A}$, следовательно, $\mathcal{A}$ - слетающий оператор. Предложение доказано.

ДоказАТЕЛЬСтво теоремы 2.1. а) Сплетающий оператор $\mathcal{A}$ может переводить подпространство $\mathcal{R}^{n, l}$ поверхностных сферических гармоник степени $l$ только в себя в силу простоты спектра квазирегулярного представления $T$ группы $\mathrm{SO}(n)$ (теорема 1.1). Тогда по лемме Шура имеем

$$
\left.\mathcal{A}\right|_{\mathcal{R}^{n, l}}=\lambda_{n, l} E
$$

где $E$ - тождественный оператор. В частности, независимо от мультииндекса $K=\left(m_{1}, m_{2}, \ldots, m_{n-3}, \pm m_{n-2}\right)$ такого, что $l \geqslant m_{1} \geqslant \cdots \geqslant m_{n-3} \geqslant m_{n-2} \geqslant 0$ имеем

$$
\left[\mathcal{A} S_{K}^{l}\right](\eta)=\int_{S^{n-1}(\xi)} F[(\xi, \eta)] S_{K}^{l}(\xi) d \xi=\lambda_{n, l} S_{K}^{l}(\eta) .
$$

Действительно, как отмечалось выше, поверхностные сферические гармоники $S_{K}^{l}(\xi)$ образуют ортогональный базис неприводимого $\operatorname{SO}(n)$-модуля $\mathcal{R}^{n, l}$.

б) Вычислим теперь собственное значение $\lambda_{n, l}$ оператора $\mathcal{A}$. Для облегчения вычислений мы можем заметить следующее. Поскольку собственное значение $\lambda_{n, l}$ не зависит от мультииндекса $K$, то можно положить $K=(0,0, \ldots, 0)$, т. е. вместо произвольной сферической гармоники рассмотреть зональную сферическую гармонику $S_{O}^{l}(\xi)$ [7, с. 437]:

$$
S_{O}^{l}(\xi)=\frac{C_{l}^{\frac{n-2}{2}}\left(\xi_{n}\right)}{C_{l}^{\frac{n-2}{2}}(1)}
$$

где $\xi=\left(\xi_{1}, \xi_{2}, \ldots, \xi_{n}\right)$ - декартовы координаты, $\xi \in S^{n-1}$.

Пусть

$$
k(\alpha)=\frac{1}{2 \pi} \int_{-1}^{1} e^{-i \alpha \beta} F(\beta) d \beta .
$$

Согласно касающимся функции $F(x)$ условиям теоремы и теореме Винера-Пэли функция $k(\alpha)$ квадратично интегрируема по Лебегу на $(-\infty,+\infty)$, а на каждом конечном интервале абсолютно интегрируема. Пусть, далее,

$$
F_{A}(\beta)=\int_{-A}^{A} e^{i \alpha \beta} k(\alpha) d \alpha .
$$

Тогда если $\beta \in[-1,1]$, то $F_{A}(\beta) \rightarrow F(\beta)$ в среднем квадратическом при $A \rightarrow \infty$, а если $\beta \notin[-1,1]$, то $F_{A}(\beta) \rightarrow 0$ при $A \rightarrow \infty$. Пусть угол между векторами $\xi$ и $\eta$ 
равен $\gamma$; тогда $(\xi, \eta)=|\xi||\eta| \cos \gamma=\cos \gamma$, так как оба вектора имеют длину 1. Итак, мы имеем

$$
\lim _{A \rightarrow \infty} F_{A}(\cos \gamma)=F(\cos \gamma)
$$

откуда

$$
\begin{aligned}
{\left[\mathcal{A} S_{O}^{l}\right](\eta) } & =\lim _{A \rightarrow \infty} \int_{S^{n-1}(\xi)} F_{A}(\cos \gamma) S_{O}^{l}(\xi) d \xi \\
& =\lim _{A \rightarrow \infty} \int_{S^{n-1}(\xi)} S_{O}^{l}(\xi) \int_{-A}^{A} e^{i \alpha \cos \gamma} k(\alpha) d \alpha d \xi \\
& =\lim _{A \rightarrow \infty} \int_{-A}^{A} k(\alpha) \int_{S^{n-1}(\xi)} e^{i \alpha \cos \gamma} S_{O}^{l}(\xi) d \xi d \alpha
\end{aligned}
$$

Воспользуемся известным разложением функции $e^{i \alpha \cos \gamma}$ (волновой функции плоской волны) в ряд по сферическим волнам $[14, \S 11$, п. 5], [7, с. 520, (2)]:

$$
e^{i \alpha \cos \gamma}=\left(\frac{2}{\alpha}\right)^{\frac{n-2}{2}} \Gamma\left(\frac{n-2}{2}\right) \sum_{m=0}^{\infty} i^{m}\left(m+\frac{n}{2}-1\right) C_{m^{\frac{n-2}{2}}}^{(\cos \gamma) J_{m+\frac{n}{2}-1}(\alpha) .}
$$

Но все сферические гармоники $C_{m}^{\frac{n-2}{2}}, m=0,1,2, \ldots$, будут ортогональны зональной сферической гармонике $S_{O}^{l}(\xi)$, за исключением одной, когда $m=l$ :

$$
\int_{S^{n-1}(\xi)} C_{m}^{\frac{n-2}{2}}[(\xi, \eta)] S_{O}^{l}(\xi) d \xi= \begin{cases}0, & \text { если } l \neq m, \\ \frac{4 \pi^{\frac{n}{2}}}{(2 l+n-2) \Gamma\left(\frac{n-2}{2}\right)} S_{O}^{l}(\eta), & \text { если } l=m .\end{cases}
$$

Для доказательства равенства (2.7) заметим, что вектор $\eta$ можно считать равным $(0,0, \ldots, 0,1)$ (иначе мы бы выбрали новый ортонормированный базис в $\left.\mathbb{R}^{n}\right)$. Тогда $(\xi, \xi)=\xi_{n}$, но и $S_{O}^{l}(\xi)$ фактически зависит только от последней координаты $\xi_{n}$ точки $\xi$, поэтому

$$
\int_{S^{n-1}(\xi)} C_{m}^{\frac{n-2}{2}}[(\xi, \eta)] S_{O}^{l}(\xi) d \xi=\frac{\mu\left(S^{n-2}\right)}{C_{l}^{\frac{n-2}{2}}(1)} \int_{-1}^{1} C_{m^{\frac{n-2}{2}}}\left(\xi_{n}\right) C_{l}^{\frac{n-2}{2}}\left(\xi_{n}\right)\left(1-\xi_{n}^{2}\right)^{\frac{n-3}{2}} d \xi_{n} .
$$

Мы воспользовались (2.5), а также тем, что инвариантная мера $d \xi$ (см. $\S 1$ ) на сфере $S^{n-1}$ выражается через $\xi_{n}$ и инвариантную меру $d \xi^{\prime}$ на сфере $S^{n-2}$ по формуле

$$
d \xi=\left(1-\xi_{n}^{2}\right)^{\frac{n-3}{2}} d \xi^{\prime} d \xi_{n} .
$$

Далее в силу соотношений ортогональности для многочленов Гегенбауэра $[15$, с. $563,(10)]$ имеем

$$
\begin{aligned}
\int_{-1}^{1} C_{m}^{\frac{n-2}{2}}\left(\xi_{n}\right) C_{l}^{\frac{n-2}{2}}\left(\xi_{n}\right)\left(1-\xi_{n}^{2}\right)^{\frac{n-3}{2}} d \xi_{n} \\
\quad= \begin{cases}0, & \text { если } m \neq l, \\
\frac{2 \sqrt{\pi}(n+l-3) !}{(n-3) ! l !(2 l+n-2)} \frac{\Gamma\left(\frac{n-1}{2}\right)}{\Gamma\left(\frac{n-2}{2}\right)}, & \text { если } m=l .\end{cases}
\end{aligned}
$$


Отсюда мы сразу получаем равенство (2.7), учитывая, что

$$
C_{l}^{\frac{n-2}{2}}(1)=\frac{(n+l-3) !}{l !(n-3) !}, \quad S_{O}^{l}(\eta)=1 .
$$

Используя соотношения ортогональности (2.7), мы можем вычислить внутренний интеграл в формуле (2.6):

$$
\begin{gathered}
\int_{S^{n-1}(\xi)} e^{i \alpha \cos \gamma} S_{O}^{l}(\xi) d \xi=\left(\frac{2}{\alpha}\right)^{\frac{n-2}{2}} \Gamma\left(\frac{n-2}{2}\right) i^{l}\left(l+\frac{n}{2}-1\right) \\
\times J_{l+\frac{n}{2}-1}(\alpha) \frac{2 \pi^{\frac{n}{2}}}{\left(l+\frac{n}{2}-1\right) \Gamma\left(\frac{n-2}{2}\right)} S_{O}^{l}(\eta) \\
=(2 \pi)^{\frac{n}{2}} i^{l} \alpha^{-\frac{n-2}{2}} J_{l+\frac{n}{2}-1}(\alpha) S_{O}^{l}(\eta) .
\end{gathered}
$$

Следовательно,

$$
\left[\mathcal{A} S_{O}^{l}\right](\eta)=\left((2 \pi)^{\frac{n}{2}} i^{l} \lim _{A \rightarrow \infty} \int_{-A}^{A} \alpha^{-\frac{n-2}{2}} J_{l+\frac{n}{2}-1}(\alpha) k(\alpha) d \alpha\right) S_{O}^{l}(\eta) .
$$

Функция $k(\alpha)$ квадратично интегрируема по Лебегу на $(-\infty,+\infty)$, это же справедливо и для $\alpha^{-\frac{n-2}{2}} J_{l+\frac{n}{2}-1}(\alpha)$. Однако произведение двух квадратично интегрируемых функций будет квадратично интегрируемо согласно неравенству Коши-Буняковского. Следовательно, несобственный интеграл в правой части равенства (2.8) сходится. Окончательно имеем

$$
\lambda_{n, l}=(2 \pi)^{\frac{n}{2}} i^{l} \int_{-\infty}^{+\infty} t^{\frac{2-n}{2}} J_{l+\frac{n}{2}-1}(t) f(t) d t,
$$

где

$$
f(t)=\frac{1}{2 \pi} \int_{-1}^{1} e^{-i x t} F(x) d x
$$

Теорема доказана.

ЗАмечАниЕ 1. Несмотря на внешнее различие формулировок задачи Минковского и теоремы Функа-Гекке, существенной (и решающей) частью обоих доказательств явилась простота спектра квазирегулярного представления $T$ группы Ли $\mathrm{SO}(n)$ в $L^{2}\left(S^{n-1}\right)$.

\section{§ 3. Обобщенная теорема Функа-Гекке}

Объектом исследований в этом параграфе будет гиперболическое пространство $\mathbb{R}^{n-1,1}$ с индефинитным скалярным произведением

$$
[x, y]=-x_{1} y_{1}-\cdots-x_{n-1} y_{n-1}+x_{n} y_{n} .
$$

Это скалярное произведение может быть использовано для определения расстояния $r(x, y)$ между двумя точками $x, y \in \mathbb{R}^{n-1,1}$, не принадлежащими световому конусу $[z, z]=0$. Положим для таких точек

$$
\operatorname{ch} r(x, y)=\frac{[x, y]}{\sqrt{[x, x] \cdot[y, y]}}
$$


Расстояние, введенное таким образом, может принимать как действительные неотрицательные, так и чисто мнимые значения. Легко показать, что если мы ограничимся внутренностью $U$ верхней полы светового конуса:

$$
U=\left\{x \in \mathbb{R}^{n-1,1} \mid[x, x]>0, x_{n}>0\right\},
$$

то для любых двух точек $x, y \in U$ расстояние между ними будет неотрицательно. Действительно, точки, расположенные на одном и том же луче, выходящем из начала координат (и только такие точки), будут удалены друг от друга на нулевое расстояние: если $x \in U$ и $\lambda>0$, то $r(x, \lambda x)=0$.

В области $U$ определим “гиперболические координаты”, аналогичные сферическим координатам из $\S 1$ :

$$
\begin{gathered}
x_{1}=r \operatorname{sh} \theta_{n-1} \sin \theta_{n-2} \cdots \sin \theta_{2} \sin \theta_{1}, \\
x_{2}=r \operatorname{sh} \theta_{n-1} \sin \theta_{n-2} \cdots \sin \theta_{2} \cos \theta_{1}, \\
\ldots \ldots \cdots \cdots \cdots \cdots \cdots \cdots \cdots \cdots \cdots \cdots \cdots \cdots \\
x_{n-1}=r \operatorname{sh} \theta_{n-1} \cos \theta_{n-2}, \\
x_{n}=r \operatorname{ch} \theta_{n-1},
\end{gathered}
$$

где $r=\sqrt{[x, y]}$ - расстояние от точки $x \in \mathbb{R}^{n-1,1}$ до начала координат. Здесь координаты $r, \theta_{1}, \theta_{2}, \ldots, \theta_{n-1}$ могут меняться в следующих диапазонах значений: $0 \leqslant r<\infty, 0 \leqslant \theta_{1}<2 \pi, 0 \leqslant \theta_{i}<\pi, 1<i<n-1 ; 0 \leqslant \theta_{n-1}<\infty$.

Назовем псевдосферой радиуса $R$ множество всех точек $U$, для которых $[x, x]=R^{2}$. Мы будем всюду далее использовать обозначения $S_{H}(R)$ для псевдосферы радиуса $R$ и $S_{H}$ для псевдосферы радиуса 1 в $\mathbb{R}^{n-1,1}$. Отметим, что $S_{H}$ является многообразием постоянной отрицательной кривизны в $\mathbb{R}^{n-1,1}$, называемым $(n-1)$-мерным пространством Лобачевского.

Каждая псевдосфера $S_{H}$ радиуса $R$ является однородным пространством относительно действия группы $G=\mathrm{SO}_{0}(n-1,1)$, где индекс 0 обозначает взятие связной компоненты единицы группы. Поскольку группа $G$ унимодулярна, то факторпространство $\mathrm{SO}_{0}(n-1,1) / \mathrm{SO}(n-1) \cong S_{H}$ обладает единственной с точностью до постоянного множителя инвариантной относительно $G$ мерой $d \xi$ (см., например, [16, с. 163]), которая в гиперболических координатах (3.1) принимает вид

$$
d \xi=\operatorname{sh}^{n-2} \theta_{n-1} \cdot \sin ^{n-3} \theta_{n-2} \cdots \sin \theta_{2} d \theta_{1} d \theta_{2} \ldots d \theta_{n-1} .
$$

Пусть $L_{\text {loc }}^{1}\left(S_{H}^{n-1}, d \xi\right)$ - пространство локально интегрируемых ${ }^{3}$ по мере $d \xi$ комплексных функций на $S_{H}$. Отметим очевидные включения [17, с. 121]

$$
L_{\mathrm{loc}}^{1}\left(S_{H}, d \xi\right) \supset L_{\mathrm{loc}}^{2}\left(S_{H}, d \xi\right) \supset L^{2}\left(S_{H}, d \xi\right) .
$$

В пространстве $L_{\text {loc }}^{1}\left(S_{H}, d \xi\right)$ действует квазирегулярное представление $R$ группы Ли $\mathrm{SO}_{0}(n-1,1)$, определяемое равенством

$$
(R(g) f)(x)=f\left(g^{-1} x\right), \quad f \in L_{\mathrm{loc}}^{1}\left(S_{H}, d \xi\right) .
$$

\footnotetext{
${ }^{3}$ Как обычно, мы отождествляем функции $f(x), g(x) \in L_{\mathrm{loc}}^{1}\left(S_{H}^{n-1}, d \xi\right)$, если они отличаются друг от друга лишь на множестве меры нуль.
} 
Действительно, для любого компакта $K \subset S_{H}$ имеем

$$
\int_{K}|(R(g) f)(\xi)| d \xi=\int_{K}\left|f\left(g^{-1} \xi\right)\right| d \xi=\int_{K^{\prime}}|f(\eta)| d \eta<\infty,
$$

где положено $g^{-1} K=K^{\prime}$. Хорошо известно [18], что квазирегулярное представление $R$ имеет следующее разложение в прямой интеграл неприводимых представлений группы $G$ :

$$
R=\int_{0}^{\infty} R^{\sigma} d \rho, \quad \sigma=-\frac{n-2}{2}+i \rho, \quad \rho \in[0,+\infty) .
$$

Отметим, что все представления $R^{\sigma}$ бесконечномерны. В пространстве $L_{\mathrm{loc}}^{1}\left(S_{H}^{n-1}, d \xi\right)$, где $\sigma$ принимает те же значения, что и в $(3.3)$, рассмотрим функции

$$
\begin{aligned}
H_{L}^{n \sigma}\left(\theta_{1}, \ldots, \theta_{n-1}\right)=\operatorname{sh}^{\frac{3-n}{2}} \theta_{n-1} \mathfrak{P}_{\sigma+\frac{3-3}{2}}^{\frac{3-n}{2}-k_{0}}\left(\operatorname{ch} \theta_{n-1}\right) \\
\quad \times \prod_{j=0}^{n-4} C_{k_{j}-k_{j+1}}^{\frac{n-j-3}{2}+k_{j+1}} \cos \left(\theta_{n-j-2}\right) \sin ^{k_{j+1}}\left(\theta_{n-j-2}\right) e^{ \pm i k_{n-3} \theta_{1}}
\end{aligned}
$$

где $\mathfrak{P}_{\nu}^{\mu}(x)$ - присоединенные функции Лежандра первого рода, $C_{\nu}^{\mu}(x)-$ многочлены Гегенбауэра, $L=\left(k_{0}, k_{1}, \ldots, k_{n-4}, \pm k_{n-3}\right), k_{0} \geqslant k_{1} \geqslant \cdots \geqslant k_{n-3} \geqslant 0$, и все параметры $k_{i}$ целые. Можно проверить [7, с. 517], что если продолжить функции $H_{L}^{n \sigma}(\theta)$ с псевдосферы $S_{H}$ на $U$ "по однородности" со степенью однородности $\sigma$, то полученные функции $\widetilde{H}_{L}^{n \sigma}(\theta)$ на $U$ будут решениями волнового уравнения $\square \widetilde{H}_{L}^{n \sigma}=0$, т. е. пространственными гиперболическими гармониками. Таким образом, мы можем назвать $H_{L}^{n \sigma}(\theta)$ поверхностными гиперболическими гармониками и считать их аналогами поверхностных сферических гармоник $S_{k}^{l}(\theta)$ из $\S 2$.

Далее будем записывать поверхностные гиперболические гармоники в виде

$$
H_{L}^{n \sigma}\left(\theta_{1}, \ldots, \theta_{n-1}\right)=\operatorname{sh}^{\frac{3-n}{2}}\left(\theta_{n-1}\right) \mathfrak{P}_{\sigma+\frac{3-n}{2}}^{\frac{3-n}{2}}\left(\operatorname{ch} \theta_{n-1}\right) S_{K}^{k_{0}}\left(\theta_{1}, \ldots, \theta_{n-2}\right),
$$

где $S_{K}^{k_{0}}(\theta)$ - поверхностные сферические гармоники на $(n-2)$-мерной сфере $S^{n-2}($ см. $(2.1)), L=\left(k_{0}, K\right), K=\left(k_{1}, \ldots, k_{n-4}, \pm k_{n-3}\right)$, причем $k_{0} \geqslant k_{1} \geqslant \cdots$ $\cdots \geqslant k_{n-3} \geqslant 0$.

ТЕОРема 3.1. Поверхностные гиперболические гармоники $H_{L}^{n \sigma}(\theta)$ образуют "непрерывныи" базис пространства $L_{\mathrm{loc}}^{1}\left(S_{H}\right)$.

ДокАзАТЕЛЬство. Покажем сначала, что все гармоники $H_{L}^{n \sigma}(\theta)$ при $\sigma=$ $-\frac{n-2}{2}+i \rho, \rho \in[0,+\infty)$, и всех значениях мультииндекса $L$ линейно независимы $($ над $\mathbb{C})$. Гармоники, отличающиеся друг от друга лишь сферической частью, линейно независимы, так как они ортогональны друг другу на каждой сфере, получаемой пересечением гиперболоида $S_{H}$ с гиперплоскостью $x=$ const $>1$. Следовательно, они ортогональны и на $S_{H}$. Далее, гармоники, отвечающие различным значениям $\sigma$, линейно независимы ввиду того, что преобразование Гельфанда-Граева сопоставляет функции $H_{L}^{n \sigma}(\theta)$ функцию $\Phi(y, \sigma)$ на верхней 
поле́ конуса $[z, z]=0$, имеющую по $y$ степень однородности $\sigma$ [7, гл. $\mathrm{X}, \S 3,4]$. Осталось доказать, что линейно независимы гармоники $H_{L}^{n \sigma}(\theta)$, отличающиеся друг от друга лишь значениями параметра $k_{0}$. Как показано в работе [19, c. $239,(13)$ и с. $228,(1)]$, параметр $k_{0}$ отвечает за номер строки в одном и том же столбце матрицы оператора $T^{n \sigma}(g)$ группы $G$. Но матричные элементы неприводимого представления полупростой группы Ли всегда линейно независимы.

Докажем теперь, что всякая функция $f(\theta) \in L_{\mathrm{loc}}^{1}\left(S_{H}\right)$ представима в виде

$$
f(\theta)=\sum_{L} \int_{0}^{+\infty} a_{L}^{n \sigma}(\theta) H_{L}^{n \sigma}(\theta) d \rho .
$$

Предположим от противного, что существует такая ненулевая функция $f(\theta) \in$ $L_{\mathrm{loc}}^{1}\left(S_{H}\right)$, что для любой поверхностной гиперболической гармоники $H_{L}^{n \sigma}(\theta)$ выполнено

$$
\int_{S_{H}} f(\theta) \overline{H_{L}^{n \sigma}(\theta)} d \theta=0,
$$

где $d \theta$ совпадает с инвариантной мерой (3.2). Запишем интегрирование по $S_{H}$ как повторное интегрирование по $\theta_{n-1} \in[0,+\infty)$ и по сфере $S^{n-2}\left(\theta_{n-1}\right)$, получаемой пересечением гиперболоида $S_{H}$ с гиперплоскостью $x_{n}=\operatorname{ch} \theta_{n-1}$ :

$$
\begin{aligned}
\int_{0}^{+\infty} \operatorname{sh}^{\frac{n-1}{2}}\left(\theta_{n-1}\right) \overline{\mathfrak{P}_{\sigma+\frac{n-3}{2}}^{\frac{3-n}{2}-k_{0}}\left(\operatorname{ch} \theta_{n-1}\right)}\left(\int_{S^{n-2}\left(\theta_{n-1}\right)} f(\theta)\right. \\
\left.\quad \times \overline{S_{K}^{k_{0}}\left(\theta_{1}, \ldots, \theta_{n-2}\right)} \sin ^{n-3} \theta_{n-2} \cdots \sin \theta_{2} d \theta_{1} \ldots d \theta_{n-2}\right) d \theta_{n-1}=0 .
\end{aligned}
$$

Функцию, стоящую в равенстве $(3.4)$ в скобках, обозначим через $p\left(\theta_{n-1}\right)$. Ясно, что $p\left(\theta_{n-1}\right) \in L_{\text {loc }}^{1}(0,+\infty)$. Функцию, стоящую в равенстве $(3.4)$ перед скобками, при замене переменной $x=\operatorname{ch} \theta_{n-1}$ можно привести к виду $\left(x^{2}-1\right)^{\frac{n-1}{4}} \mathfrak{P}_{-\frac{1}{2}+i \rho}^{\frac{3-n}{2}-k_{0}}(x)$. Легко проверить, что для всех значений $k_{0}$ и $\rho$, указанных выше, эти функции являются собственными функциями некоторого самосопряженного оператора второго порядка. Применяя стандартный прием разложения по собственным функциям самосопряженных операторов, мы получаем, что любая функция $u(x)$ такая, что $\int_{1}^{+\infty}|u(x)|^{2} d x<\infty$, допускает разложение:

$$
u(x)=\int_{0}^{+\infty} a(\rho)\left(x^{2}-1\right)^{\frac{n-1}{4}} \mathfrak{P}_{\frac{1}{2}+i \rho}^{\frac{3-n}{2}-k_{0}}(x) d \rho
$$

(cp. c $[7$, c. $326,(6)])$.

Теперь (3.4) можно переписать в виде

$$
\int_{0}^{+\infty} \overline{u\left(\theta_{n-1}\right)} p\left(\theta_{n-1}\right) d \theta_{n-1}=0
$$

причем равенство нулю справедливо для всех $u(x) \in L^{2}(0,+\infty)$, а значит, и для всех $u\left(\theta_{n-1}\right) \in C_{0}^{\infty}(0,+\infty)$. Согласно теореме из $[17$, с. 122$]$ имеем $p\left(\theta_{n-1}\right)=0$ почти всюду на $(0,+\infty)$. Это означает, что для почти всех $\theta_{n-1} \in(0,+\infty)$ 
и всех поверхностных сферических гармоник $S_{K}^{k_{0}}(\theta)$ справедливо равенство

$$
\begin{aligned}
\int_{S^{n-2}\left(\theta_{n-1}\right)} f\left(\theta_{1}, \ldots, \theta_{n-2}, \theta_{n-1}\right) \overline{S_{K}^{k_{0}}\left(\theta_{1}, \ldots, \theta_{n-2}\right)} \\
\times \sin ^{n-3} \theta_{n-2} \cdots \sin \theta_{2} d \theta_{1} \ldots d \theta_{n-2}=0 .
\end{aligned}
$$

Зафиксируем произвольное значение $\theta_{n-1}$, для которого это равенство справедливо. Хорошо известно, что поверхностные сферические гармоники $S_{K}^{k_{0}}(\theta)$ образуют базис в пространстве всех многочленов на сфере $S^{n-2}\left(\theta_{n-1}\right)$ (см., например, [7, с. 426] или [19, с. 160, следствие 2.2]). Согласно теореме Вейерштрасса пространство многочленов на $S^{n-2}\left(\theta_{n-1}\right)$ плотно в пространстве $C\left(S^{n-2}\left(\theta_{n-1}\right)\right)$ непрерывных функций на сфере. Далее, из С-свойства измеримых функций Лузина (см., например, [20, теорема 4]) следует, что всякая ограниченная измеримая функция на сфере есть предел в среднем последовательности непрерывных функций. Поэтому пространство $C\left(S^{n-2}\left(\theta_{n-1}\right)\right)$ плотно в пространстве $L^{\infty}\left(S^{n-2}\left(\theta_{n-1}\right)\right)$ ограниченных измеримых функций.

Итак, мы имеем

$$
\begin{aligned}
\int_{S^{n-2}\left(\theta_{n-1}\right)} f( & \left.\theta_{1}, \ldots, \theta_{n-2}, \theta_{n-1}\right) \overline{g\left(\theta_{1}, \ldots, \theta_{n-2}\right)} \\
& \times \sin ^{n-3} \theta_{n-2} \cdots \sin \theta_{2} d \theta_{1} \ldots d \theta_{n-2}=0
\end{aligned}
$$

для любой ограниченной измеримой на сфере функции $g$. Однако, как известно, для любого компакта $K$ в $\mathbb{R}^{n}$ справедливо

$$
L^{\infty}(K)=\left(L^{1}(K)\right)^{*},
$$

причем линейные функционалы из $\left(L^{p}(K)\right)^{*}$ разделяют элементы из $L^{p}(K)$, $p \geqslant 1\left[17\right.$, теорема 2.10], поэтому (3.5) возможно лишь для функции $f\left(\theta_{1}, \ldots\right.$ $\left.\ldots, \theta_{n-2}, \theta_{n-1}\right)=0$ почти всюду на сфере $S^{n-2}\left(\theta_{n-1}\right)$. Следовательно, $f\left(\theta_{1}, \ldots, \theta_{n-2}, \theta_{n-1}\right)=0$ для почти всех точек из $S_{H}$. Поскольку мы отождествляем функции из $L_{\text {loc }}^{1}\left(S_{H}\right)$, отличающиеся друг от друга лишь на множестве меры нуль, то полученное равенство нулю противоречит нашему предположению. Теорема доказана.

Обозначим через $\mathcal{H}^{n \sigma}$ замыкание в $L_{\text {loc }}^{1}\left(S_{H}\right)$ линейной оболочки всех поверхностных гиперболических гармоник $H_{L}^{n \sigma}(\theta)$ степени однородности $\sigma$.

ТеОРема 3.2. Пространство $\mathcal{H}^{\text {пб } я в л я е т с я ~ п р о с т р а н с т в о м ~ н е п р и в о д и м о-~}$ го представления группъ Ли $\mathrm{SO}_{0}(n-1,1)$.

ДокАЗАТЕЛЬСтво. Покажем, что $\mathcal{H}^{n \sigma}$ инвариантно относительно операторов квазирегулярного представления $R(g)$. Очевидно, сомнение может вызвать лишь гиперболический поворот $g \in \mathrm{SO}_{0}(n-1,1)$. Без ограничения общности можно считать $g$ поворотом в осях координат $x_{n-1}$ и $x_{n}$. Легко видеть, что инфинитезимальным оператором такого поворота является оператор $X=-\frac{\partial}{\partial \theta_{n-1}}$. Используя обычные свойства функций Лежандра (см., например, [15, с. 775 и 778]), можно показать, что: 
a) при $k_{0}=0$

$$
\frac{\partial}{\partial \theta_{n-1}} H_{(0, K)}^{n \sigma}(\theta)=\sigma(\sigma+n-2) H_{(1, K)}^{n \sigma}(\theta)
$$

б) при $k_{0} \geqslant 1$

$$
\begin{gathered}
\frac{\partial}{\partial \theta_{n-1}} H_{\left(k_{0}, K\right)}^{n \sigma}(\theta)=\frac{\left[\left(n+k_{0}-3\right)\left(\sigma-k_{0}\right)-k_{0}\right]\left(\sigma+n+k_{0}-2\right)}{n+2 k_{0}-3} H_{\left(k_{0}+1, K\right)}^{n \sigma}(\theta) \\
+\frac{k_{0}}{n+2 k_{0}-3} H_{\left(k_{0}-1, K\right)}^{n \sigma}(\theta)
\end{gathered}
$$

Таким образом, $\mathcal{H}^{n \sigma}$ инвариантно относительно $X$, а значит, и относительно всех операторов $R(g)$. Неприводимость $\mathcal{H}^{n \sigma}$ доказана в [7, гл. Х, § 2.3]. Теорема доказана.

СлЕДСтвиЕ 3.1. Квазирегулярное представление $R$ группъ Ли $\mathrm{SO}_{0}(n-1,1)$ в пространстве $L_{\mathrm{loc}}^{1}\left(S_{H}, d \xi\right)$ имеет простой спектр.

ДокАЗАТЕЛЬСтво. Волновой оператор

$$
\square=\frac{\partial^{2}}{\partial x_{1}^{2}}+\cdots+\frac{\partial^{2}}{\partial x_{n-1}^{2}}-\frac{\partial^{2}}{\partial x_{n}^{2}}
$$

в гиперболических координатах (3.1) представляется в виде $\square=\square_{r}+\square_{\theta}$, где

$$
\square_{r}=-\frac{1}{r^{n-1}} \frac{\partial}{\partial r} r^{n-1} \frac{\partial}{\partial r}
$$

- радиальная часть, а

$$
\square_{\theta}=\frac{1}{r^{2} \operatorname{sh}^{n-2}\left(\theta_{n-1}\right)} \frac{\partial}{\partial \theta_{n-1}} \operatorname{sh}^{n-2}\left(\theta_{n-1}\right) \frac{\partial}{\partial \theta_{n-1}}+\cdots
$$

- угловая часть; здесь многоточие заменяет члены, содержащие дифференцирования по сферическим координатам $\theta_{n-2}, \ldots, \theta_{1}$, которые нам сейчас не понадобятся. Точное выражение для $\square_{\theta}$ приведено в [7, с. 517]. Поскольку $S_{H}=\mathrm{SO}_{0}(n-1,1) / \mathrm{SO}(n-1)$ является симметрическим пространством ранга 1 , то всякий $G$-инвариантный дифференциальный оператор на $S_{H}$ является многочленом от оператора Казимира второго порядка группы $G$, который на $S_{H}$ совпадает (с точностью до константы) с оператором Лапласа-Бельтрами, т. е. $\square_{\theta}$ (см. [21, гл. 10]). Но оператор Казимира по определению является элементом центра $U(\mathfrak{g})$, где $\mathfrak{g}$ - алгебра Ли группы $G$. Поэтому $\square_{\theta}$ коммутирует со всеми операторами $R(g)$ квазирегулярного представления $R$ группы $G$.

Теперь, если окажется, что представления $G$ в пространствах $\mathcal{H}^{n \sigma}$ и $\mathcal{H}^{n \sigma^{\prime}}$ при $\sigma \neq \sigma^{\prime}$ изоморфны, то оператор $\square_{\theta}$ на этих пространствах обязан иметь один и тот же спектр. Вычислим спектр $\square_{\theta}$ на пространстве $\mathcal{H}^{n \sigma}$. Без ограничения общности возьмем в качестве "тестовой” функции зональную гиперболическую гармонику

$$
H_{(0,0)}^{n \sigma}\left(\theta_{n-1}\right)=\operatorname{sh}^{\frac{3-n}{2}}\left(\theta_{n-1}\right) \mathfrak{P}_{\sigma+\frac{n-3}{2}}^{\frac{3-n}{2}}\left(\operatorname{ch} \theta_{n-1}\right) .
$$


Используя (3.6) и заменяя для краткости $\theta_{n-1}$ на $\theta$, получим

$$
\begin{aligned}
\square_{\theta} H_{(0,0)}^{n \sigma}(\theta)= & \frac{\sigma(\sigma+n-2)}{\operatorname{sh}^{n-2}(\theta)} \frac{\partial}{\partial \theta}\left(\operatorname{sh}^{\frac{n-1}{2}}(\theta) \mathfrak{P}_{\sigma+\frac{n-3}{2}}^{\frac{1-n}{2}}(\operatorname{ch} \theta)\right) \\
= & \sigma(\sigma+n-2)\left((n-1) \operatorname{sh}^{\frac{1-n}{2}}(\theta) \operatorname{ch}(\theta) \mathfrak{P}_{\sigma+\frac{n-3}{2}}^{\frac{1-n}{2}}(\operatorname{ch} \theta)\right. \\
& \left.\quad+(\sigma-1)(\sigma+n-1) \operatorname{sh}^{\frac{3-n}{2}}(\theta) \mathfrak{P}_{\sigma+\frac{n-3}{2}}^{-\frac{n+1}{2}}(\operatorname{ch} \theta)\right) .
\end{aligned}
$$

Выражение в скобках можно упростить, используя рекуррентное соотношение для функций Лежандра [15, с. 775]. Тогда

$$
\square_{\theta} H_{(0,0)}^{n \sigma}(\theta)=\sigma(\sigma+n-2) \operatorname{sh}^{\frac{3-n}{2}}(\theta) \mathfrak{P}_{\sigma+\frac{n-3}{2}}^{\frac{3-n}{2}}(\operatorname{ch} \theta)=-\left(\rho^{2}+\left(\frac{n-2}{2}\right)^{2}\right) H_{(0,0)}^{n \sigma}(\theta) .
$$

Таким образом, представления $G$ в пространствах $\mathcal{H}^{n \sigma}$ и $\mathcal{H}^{n \sigma^{\prime}}$ при $\sigma \neq \sigma^{\prime}$ могут быть изоморфны лишь в случае, когда $\sigma=-\frac{n-2}{2}+i \rho$ и $\sigma^{\prime}=-\frac{n-2}{2}-i \rho$. Однако в $L_{\text {loc }}^{1}\left(S_{H}, d \xi\right)$ согласно теореме 3.1 базис образуют функции $H_{L}^{n \sigma}(\theta)$, где $\sigma=-\frac{n-2}{2}+i \rho$ и $\rho \in[0,+\infty)$, т. е. указанный случай сопряженных значений $\sigma$ невозможен. Следствие доказано.

Итак, “прямое слагаемое” $R^{\sigma}$ квазирегулярного представления $R$ в (3.3) действует в точности в пространстве $\mathcal{H}^{n \sigma}$ поверхностных гиперболических гармоник степени однородности $\sigma$.

В работе [22] было получено следующее обобщение теоремы Функа-Гекке на случай гиперболического пространства $\mathbb{R}^{n-1,1}$, также использующее свойства сплетающих операторов.

ТЕОРема 3.3. Пусть функиия $F(x)$ действительного переменного такова, чmo:

(a) $F(x) \in L^{1}(-\infty,+\infty) \cap L^{2}(-\infty,+\infty)$;

(b) $F(x)$ допускает аналитическое продолжение до функиии $F(x+i y)$ комплексного переменного $x+i y$, ограниченной и аналитической в нижней полуплоскости $y \leqslant 0$.

Пусть $H_{L}^{n \sigma}$ - произвольная поверхностная гиперболическая гармоника степени однородности $\sigma$. Тогда для любого $\eta$, принадлежащего псевдосфере единичного радиуса, справедливо равенство

$$
\int_{S_{H}} F([\xi, \eta]) H_{L}^{n \sigma}(\xi) d \xi=\lambda_{n, \sigma} H_{L}^{n \sigma}(\eta)
$$

где собственное значение $\lambda_{n, \sigma}$ не зависит от мультииндекса $L$ гармони$\kappa u H_{L}^{n \sigma}$.

Отметим, что хотя теорема 3.3 была доказана в работе [22, теорема 2] для произвольного значения $n \in \mathbb{N}$, однако собственное значение $\lambda_{n, \sigma}$ было вычислено лишь для двух важных с физической точки зрения значений размерности: $n=2$ и $n=4$. Кроме того, в формулировке условий, которым должна удовлетворять функция $F(x)$, было указано требование экспоненциального убывания 
при $x \rightarrow \pm \infty$, которое без ущерба может быть отброшено, как следует из доказательства теоремы 3.4, приводимой ниже. Для полноты формулировки данной теоремы приведем также полученные ранее собственные значения:

$$
\lambda_{2, \sigma}=2 \int_{0}^{+\infty} f(\alpha) K_{\sigma}(i \alpha) d \alpha, \quad \lambda_{4, \sigma}=-4 \pi i \int_{0}^{+\infty} \frac{f(\alpha) K_{\sigma+1}(i \alpha)}{\alpha} d \alpha
$$

где

$$
f(\alpha)=\frac{1}{\sqrt{2 \pi}} \int_{-\infty}^{+\infty} F(t) e^{i \alpha t} d t
$$

а $K_{\nu}(t)$ - функция Макдональда.

ОПРЕДЕЛЕНИЕ 3.1. Пусть $F(x)$ - ограниченная измеримая функция. Рассмотрим интегральный оператор $\mathcal{A}$ из $L_{\mathrm{loc}}^{1}\left(S_{H}(\xi), d \xi\right)$ в $L_{\mathrm{loc}}^{1}\left(S_{H}(\eta), d \eta\right)$, определяемый равенством ${ }^{4}$

$$
[\mathcal{A} f](\eta)=\int_{S_{H}(\xi)} F([\xi, \eta]) f(\xi) d \xi
$$

Как было показано в [22, лемма 2], $\mathcal{A}$ является сплетающим оператором квазирегулярного представления $R$ группы Ли $\mathrm{SO}_{0}(n-1,1)$. Согласно следствию 3.1 представление $R$ имеет простой спектр, поэтому сплетающий оператор $\mathcal{A}$ может переводить подпространство $\mathcal{H}^{n \sigma}$ только в себя. Отсюда ввиду леммы Шура ${ }^{5}$ мы получаем равенство (3.7). Следующая теорема уточняет формулировку обобщенной теоремы Функа-Гекке (теорема 3.3 ), определяя собственное значение $\lambda_{n, \sigma}$ в (3.7) для всех $n \in \mathbb{N} \backslash\{1\}$.

Tеорема 3.4. Собственное значение $\lambda_{n, \sigma}$ интегрального оператора $\mathcal{A} в$ пространстве $\mathcal{H}^{n \sigma}$ поверхностных гиперболических гармоник степени однородности б выражается формулой

$$
\lambda_{n, \sigma}=2^{\frac{n}{2}}(-\pi i)^{\frac{n-2}{2}} \int_{0}^{+\infty} \frac{f(\alpha) K_{\sigma+\frac{n-2}{2}}(i \alpha)}{\alpha^{\frac{n-2}{2}}} d \alpha,
$$

где $K_{\nu}(z)-$ функция Макдональда, а

$$
f(\alpha)=\frac{1}{\sqrt{2 \pi}} \int_{-\infty}^{+\infty} F(t) e^{i \alpha t} d t
$$

Доказательство. а) Рассмотрим сначала важный частный случай определения 3.1 , в котором оператор $\mathcal{A}$ имеет тригонометрическое ядро вида $\Phi(x)=$

\footnotetext{
${ }^{4}$ Точнее, мы ограничиваемся рассмотрением таких $f \in L_{\text {loc }}^{1}\left(S_{H}(\xi), d \xi\right)$, для которых функция $\xi \longmapsto F([\xi, \eta]) f(\xi)$ суммируема по мере $(3.2)$ на $S_{H}(\xi)$ для почти всех $\eta \in S_{H}(\eta)$. Для указанных $f$, очевидно, справедливо $[\mathcal{A} f](\eta) \in L_{\mathrm{loc}}^{1}\left(S_{H}(\eta), d \eta\right)$.

${ }^{5}$ Нам нужна здесь не столько лемма Шура, сколько ее следствие - операторная неприводимость представления $R^{\sigma}$. Непосредственное доказательство операторной неприводимости $R^{\sigma}$ в случае группы Ли $\mathrm{SO}_{0}(3,1)$ приведено в $[18$, гл. $3, \S 5]$, общий случай рассматривается аналогично. Лемма Шура справедлива также для так называемых квазипростых представлений редуктивных групп Ли (результат Хариш-Чандры).
} 
$\cos (s x), \Psi(x)=\sin (s x)$ или, более общо, $F(x)=e^{i s x}, s \in \mathbb{R} \backslash\{0\}$. Ввиду соотношений

$$
F(x)=\Phi(x)+i \Psi(x), \quad 2 \Phi(x)=F(x)+F(-x), \quad 2 i \Psi(x)=F(x)-F(-x)
$$

достаточно рассмотреть один лишь комплексный вариант ядра. Все предыдущие рассуждения относительно инвариантности подпространств $\mathcal{H}^{n \sigma}$ будут справедливы, разумеется, и для таких операторов $\mathcal{A}$. Покажем теперь, что для любого вектора $\eta$ такого, что $[\eta, \eta]=1$ и $\eta_{n}>0$, справедливо равенство

$$
\int_{S_{H}} e^{-i s[\xi, \eta]} H_{(0,0)}^{n \sigma}(\xi) d \xi=\mu_{n}(s, \sigma) H_{(0,0)}^{n \sigma}(\eta),
$$

где

$$
\mu_{n}(s, \sigma)=2^{\frac{n}{2}}\left(-\frac{\pi i}{s}\right)^{\frac{n-2}{2}} K_{\sigma+\frac{n-2}{2}}(i s)
$$

(cp. с [22, теорема 1]).

Собственное значение $\mu(s, \sigma)$ оператора $\mathcal{A}$ на пространстве $\mathcal{H}^{n \sigma}$ не зависит от выбора вектора $\eta$, поэтому мы можем выбрать в качестве $\eta$ вершину $\eta_{0}$ верхней полы гиперболоида $[\eta, \eta]=1$. Вершина $\eta_{0} \in S_{H}$, как и "северный полюс" $\xi_{0} \in S^{n-1}$, имеет эйлеровы координаты, равные нулю: $\left(\varphi_{1}, \varphi_{2}, \ldots, \varphi_{n-1}\right)=$ $(0,0, \ldots, 0)$. Тогда

$$
[\xi, \eta]=\operatorname{ch} \theta_{n-1} \operatorname{ch} \varphi_{n-1}=\operatorname{ch} \theta_{n-1},
$$

поэтому

$$
I=\int_{S_{H}} e^{-i s[\xi, \eta]} H_{(0,0)}^{n \sigma}(\xi) d \xi=\int_{S_{H}} e^{-i s \operatorname{ch} \theta_{n-1}} \operatorname{sh}^{\frac{3-n}{2}}\left(\theta_{n-1}\right) \mathfrak{P}_{\sigma+\frac{3-3}{2}}^{\frac{3-n}{2}}\left(\operatorname{ch} \theta_{n-1}\right) d \xi,
$$

где мера $d \xi$ на $S_{H}$ выражается формулой (3.2). Разделяя “чисто гиперболические" и сферические эйлеровы координаты, получим

$$
\begin{aligned}
& I=\int_{0}^{+\infty} e^{-i s \operatorname{ch} \theta_{n-1}} \operatorname{sh}^{\frac{n-1}{2}}\left(\theta_{n-1}\right) \mathfrak{P}_{\sigma+\frac{3-3}{2}}^{\frac{3-n}{2}}\left(\operatorname{ch} \theta_{n-1}\right) \\
& \times\left(\int_{S^{n-2}} \sin ^{n-3} \theta_{n-2} \cdots \sin \theta_{2} d \theta_{1} d \theta_{2} \ldots d \theta_{n-2}\right) d \theta_{n-1} .
\end{aligned}
$$

Учитывая, что внутренний интеграл равен площади поверхности $(n-2)$-мерной сферы:

$$
\mu\left(S^{n-2}\right)=\frac{2 \pi^{\frac{n-1}{2}}}{\Gamma\left(\frac{n-1}{2}\right)}
$$

и вводя новую переменную $t=\operatorname{ch} \theta_{n-1}$, получим

$$
I=\frac{2 \pi^{\frac{n-1}{2}}}{\Gamma\left(\frac{n-1}{2}\right)} \int_{1}^{+\infty} e^{-i s t}\left(t^{2}-1\right)^{\frac{n-3}{4}} \mathfrak{P}_{\sigma+\frac{3-2}{2}}^{\frac{3-n}{2}}(t) d t=\frac{2^{\frac{3}{2}} \pi^{\frac{n}{2}-1}}{\Gamma\left(\frac{n-1}{2}\right)} \frac{K_{\sigma+\frac{n-2}{2}}(i s)}{(i s)^{\frac{n-2}{2}}} .
$$

Для вычисления интеграла мы воспользовались [15, т. 3, с. 202, (5)]. 
Вычислим теперь значение $H_{(0,0)}^{n \sigma}\left(\eta_{0}\right)$. Для этого воспользуемся формулой (14) из [23, с. 157], выражающей присоединенную функцию Лежандра $\mathfrak{P}_{\nu}^{\mu}\left(\operatorname{ch} \theta_{n-1}\right)$ через гипергеометрическую функцию:

$$
\mathfrak{P}_{\nu}^{\mu}\left(\operatorname{ch} \theta_{n-1}\right)=\frac{2^{\mu}}{\Gamma(1-\mu)}\left(\operatorname{sh} \theta_{n-1}\right)^{-\mu}{ }_{2} F_{1}\left(-\mu-\nu ; 1+\nu-\mu ; 1-\mu ; \frac{1-\operatorname{ch} \theta_{n-1}}{2}\right) .
$$

Откуда ${ }^{6}$ имеем

$$
\lim _{\eta \rightarrow \eta_{0}} H_{(0,0)}^{n \sigma}(\eta)=\frac{2^{\frac{3-n}{2}}}{\Gamma\left(\frac{n-1}{2}\right)}
$$

и теперь получаем

$$
\mu_{n}(s, \sigma)=2^{\frac{n}{2}}\left(-\frac{\pi i}{s}\right)^{\frac{n-2}{2}} K_{\sigma+\frac{n-2}{2}}(i s)
$$

Формула (3.10) полностью доказана.

б) Вычислим теперь собственное значение $\lambda_{n, \sigma}$ для функции $F(x)$, удовлетворяющей условиям а) и б) теоремы 3.4. Введем, как и в доказательстве теоремы Функа-Гекке, функцию $k(x)$, определяя ее равенством

$$
k(x)=\frac{1}{\sqrt{2 \pi}} \int_{-\infty}^{+\infty} F(t) e^{i x t} d t .
$$

Тогда в силу того же условия а) функция $k(x)$ будет интегрируема с квадратом на $(-\infty,+\infty)$, а в силу условия б) и $[22$, лемма 1] носитель $k(x)$ как функция действительного переменного будет сосредоточен на правой полупрямой. Откуда имеем $k(x) \in L^{2}(0,+\infty)$. Кроме того, по той же лемме 1 функция $F(\alpha)$ допускает представление:

$$
F(\alpha)=\lim _{A \rightarrow+\infty} \int_{0}^{A} k(x) e^{-i \alpha x} d x=\lim _{A \rightarrow+\infty} F_{A}(\alpha) .
$$

Используя это представление, а также то, что собственное значение $\lambda_{n, \sigma}$ не зависит от мультииндекса $K$ (теорема 3.3 ), получим

$$
\begin{aligned}
\int_{S_{H}} F([\xi, \eta]) H_{(0,0)}^{n \sigma}(\xi) d \xi & =\lim _{A \rightarrow+\infty} \int_{S_{H}} F_{A}([\xi, \eta]) H_{(0,0)}^{n \sigma}(\xi) d \xi \\
& =\lim _{A \rightarrow+\infty} \frac{1}{\sqrt{2 \pi}} \int_{S_{H}} H_{(0,0)}^{n \sigma}(\xi) \int_{0}^{A} k(x) e^{-i x[\xi, \eta]} d x d \xi \\
& =\lim _{A \rightarrow+\infty} \frac{1}{\sqrt{2 \pi}} \int_{0}^{A} k(x) \int_{S_{H}} e^{-i x[\xi, \eta]} H_{(0,0)}^{n \sigma}(\xi) d \xi d x \\
& =\lim _{\varepsilon \rightarrow+0} \lim _{A \rightarrow+\infty} \frac{1}{\sqrt{2 \pi}} \int_{\varepsilon}^{A} k(x) \int_{S_{H}} e^{-i x[\xi, \eta]} H_{(0,0)}^{n \sigma}(\xi) d \xi d x
\end{aligned}
$$

\footnotetext{
${ }^{6}$ Отметим, что зональные гиперболические гармоники в монографии Виленкина $[7,(4)$, c. 499] отличаются от наших числовым множителем $2^{(n-3) / 2} \Gamma\left(\frac{n-1}{2}\right)$, поэтому в обозначениях Виленкина $\lim _{\eta \rightarrow \eta_{0}} H_{(0,0)}^{n \sigma}(\eta)=1$. В то же время ясно, что собственное значение $\mu_{n}(s, \sigma)$ от нормировки гармоники $H_{(0,0)}^{n \sigma}(\eta)$ не зависит.
} 
Применим к внутреннему интегралу уже доказанное равенство (3.10):

$$
\begin{array}{rl}
\int_{S_{H}} & F([\xi, \eta]) H_{(0,0)}^{n \sigma}(\xi) d \xi \\
& =\lim _{\varepsilon \rightarrow+0} \underset{A \rightarrow+\infty}{\lim } \frac{2^{\frac{n-1}{2}}}{\sqrt{\pi}} \int_{\varepsilon}^{A} k(x)\left(-\frac{\pi i}{x}\right)^{\frac{n-2}{2}} K_{\sigma+\frac{n-2}{2}}(i x) H_{(0,0)}^{n \sigma}(\eta) d x .
\end{array}
$$

Интеграл имеет предел при $A \rightarrow+\infty$, так как и $k(x)$, и $K_{\sigma+\frac{n-2}{2}}$ ограничены при $x \rightarrow+\infty[23$, с. 322,370$]$. Поэтому

$$
\begin{array}{rl}
\int_{S_{H}} & F([\xi, \eta]) H_{(0,0)}^{n \sigma}(\xi) d \xi \\
& =\left(2^{\frac{n-1}{2}} \pi^{\frac{n-3}{2}}(-i)^{\frac{n-2}{2}} \lim _{\varepsilon \rightarrow+0} \int_{\varepsilon}^{+\infty} \frac{k(x) K_{\sigma+\frac{n-2}{2}}(i x)}{x^{\frac{n-2}{2}}} d x\right) H_{(0,0)}^{n \sigma}(\eta) .
\end{array}
$$

Далее, так как $k(x)=0$ при $x \in(-\infty, 0)$, то это же равенство справедливо и для всех производных $k^{(n)}(x)$, откуда, учитывая гладкость $k(x)$ имеем $k(x)=$ $o\left(x^{k}\right)$ при $x \rightarrow+0$ для всех натуральных $k$. Функция Макдональда $K_{\sigma+\frac{n-2}{2}}(i x)$ ограничена при $x \rightarrow+0$. Это доказано, например, в [22, с. 223]. Окончательно получаем

$$
\int_{S_{H}} F([\xi, \eta]) H_{(0,0)}^{n \sigma}(\xi) d \xi=\lambda_{n, \sigma} H_{(0,0)}^{n \sigma}(\eta),
$$

где

$$
\lambda_{n, \sigma}=2^{\frac{n}{2}}(-\pi i)^{\frac{n-2}{2}} \int_{0}^{+\infty} \frac{k(x) K_{\sigma+\frac{n-2}{2}}(i x)}{x^{\frac{n-2}{2}}} d x, \quad k(x)=\frac{1}{\sqrt{2}} \int_{-\infty}^{+\infty} F(t) e^{i x t} d t .
$$

Теорема доказана.

\section{Список литературы}

1. A. Erdélyi, "Die Funksche Integralgleichung der Kugelflächen-funktionen und ihre Übertragung auf die Überkugel", Math. Ann., 115:1 (1938), 456-465.

2. E. Hecke, "Über orthogonal-invariante Integralgleichungen", Math. Ann., 78:1-4 (1916), 398-404.

3. И. Стейн, Г. Вейс, Введение в гармонический анализ на евклидовых пространствах, Мир, М., 1974; пер. с англ.: Е. M. Stein, G. Weiss, Introduction to Fourier analysis on Euclidean spaces, Princeton Math. Ser., 32, Princeton Univ. Press, Princeton, NJ, 1971.

4. С. Хелгасон, Группы и геометрический анализ, Мир, М., 1987; пер. с англ.: S. Helgason, Groups and geometric analysis, Pure Appl. Math., 113, Academic Press, Orlando, FL, 1984.

5. В.П. Бурский, Т.В. Штепина, "О спектре эквивариантного расширения оператора Лапласа в шаре", Укр. матем. журн., 52:11 (2000), 1473-1483; англ. пер.: V.P. Burskii, T. V. Shtepina, "On the spectrum of an equivariant extension of the Laplace operator in a ball", Ukrainian Math. J., 52:11 (2000), 1679-1690.

6. А.А. Кириллов, Лекции по методу орбит, Научн. книга, Новосибирск, 2002; англ. пер.: А. A. Kirillov, Lectures on the orbit method, Grad. Stud. Math., 64, Amer. Math. Soc., Providence, RI, 2004. 
7. Н. Я. Виленкин, Специальные функиии и теория представлений групп, 2-е изд., Наука, М., 1991; англ. пер. 1-го изд.: N. Ya. Vilenkin, Special functions and the theory of group representations, Transl. Math. Monogr., 22, Amer. Math. Soc., Providence, RI, 1968.

8. Д. П. Желобенко, Компактные группы Ли и их представления, Наука, М., 1970; англ. пер.: D.P. Želobenko, Compact Lie groups and their representations, Amer. Math. Soc., Providence, RI, 1973

9. В. В. Штепин, "Промежуточная ортогональная алгебра Ли $\mathfrak{b}_{n-1 / 2}$ и ее конечномерные представления", Изв. РАН. Сер. матем., 62:3 (1998), 201-223; англ. пер.: V.V. Shtepin, "The intermediate orthogonal Lie algebra $\mathfrak{b}_{n-1 / 2}$ and its finite-dimensional representations", Izv. Math., 62:3 (1998), 627-648.

10. В.В. Штепин, "Промежуточная алгебра Ли $\mathfrak{d}_{n-1 / 2}$, весовая схема и конечномерные представления со старшим весом", Изв. РАН. Сер. матем., 68:2 (2004), 159-190; англ. пер.: V.V. Shtepin, "The intermediate Lie algebra $\mathfrak{d}_{n-1 / 2}$, the weight scheme and finite-dimensional representations with highest weight", Izv. Math., 68:2 (2004), 375-404.

11. Д. П. Желобенко, А. Штерн, Представления групn Ли, Наука, М., 1983.

12. P. Funk, "Beiträge zur Theorie der Kugelfunktionen", Math. Ann., 77:1 (1915), 136-152.

13. В.С. Владимиров, "Об интегральном уравнении, связанном со сферическими функциями”, Научные доклады высшей школь, Физ.-мат. науки, 6 (1958), $142-146$.

14. Г. Н. Ватсон, Теория бесселевых функиий, ИЛ, М., 1949; пер. с англ.: G. N. Watson, A treatise on the theory of Bessel functions, Cambridge Univ. Press, Cambridge; Macmillan, New York, 1944.

15. А. П. Прудников, Ю.А. Брычков, О.И. Маричев, Интегралы и ряды. Специальные функиии, в 3-х т., Наука, М., 1983; англ. пер.: А. P. Prudnikov, Yu. A. Brychkov, O. I. Marichev, Integrals and series. Vol. 2. Special functions, Gordon \& Breach, New York, 1988.

16. А. О. Барут, Р. Рончка, Теория представлений групп и ее приложения, т. 1, Мир, M., 1980; пер. с англ.: А. O. Barut, R. Raczka, Theory of group representations and applications, Geest \& Portig, PWN-Polish Scientific Publishers, 1977.

17. Э. Либ, М. Лосс, Анализ, Научн. книга, Новосибирск, 1998; пер. с англ.: Е. H. Lieb, M. Loss, Analysis, Grad. Stud. Math., 14, Amer. Math. Soc., Providence, RI, 1996.

18. И. М. Гельфанд, М. И. Граев, Н. Я. Виленкин, Обобщенные функиии, Вып. 5. Интегральная геометрия и связанные с ней вопросы теории представлений, Физматгиз, М., 1962; англ. пер.: I. M. Gel'fand, M. I. Graev, N. Ya. Vilenkin, Generalized functions, Vol. 5: Integral geometry and representation theory, Academic Press, New York-London, 1966.

19. Н. Я. Виленкин, "Специальные функции, связанные с представлениями класса 1 групп движений пространств постоянной кривизны", Тр. MMO, 12, Изд-во Моск. ун-та, M., 1963, 185-257; англ. пер.: N. Ya. Vilenkin, "Special functions connected with class 1 representations of groups of motions in spaces of constant curvature", Trans. Moscow Math. Soc., 12 (1963), 209-290.

20. Л. А. Люстерник, В.И. Соболев, Краткий курс функционального анализа, Высшая школа, М., 1982.

21. С. Хелгасон, Дифференциальная геометрия и симметрические пространства, Мир, М., 1964; пер. с англ.: S. Helgason, Differential geometry and symmetric spaces, Pure Appl. Math., 12, Academic Press, New York-London, 1962. 
22. Т.В. Штепина, "Обобщение теоремы Функа-Гекке на случай гиперболического пространства", Изв. РАН. Сер. матем., 68:5 (2004), 213-224; англ. пер.: T. V. Shtepina, "A generalization of the Funk-Hecke theorem to the case of hyperbolic spaces", Izv. Math., 68:5 (2004), 1051-1061.

23. А. Кратцер, В. Франц, Трансцендентные функции, ИЛ, М., 1963; пер. с нем.: A. Kratzer, W. Franz, Transzendente Funktionen, Geest \& Portig, Leipzig, 1960.

B. В. Штепин (V. V. Shtepin)

Донецкий национальный университет

E-mail: shtepina@stels.net

T. В. Штепина (T. V. Shtepina)

Донецкий институт социального образования

E-mail: asar-feuer@rambler.ru
Поступило в редакцию

17.08 .2007

26.05 .2008 